\title{
An experimental investigation into cavitation behaviour and pressure characteristics of alternative blade sections for propellers
}

\author{
Emin Korkut ${ }^{1}$, Mehmet Atlar $^{2}$ and Dazheng Wang ${ }^{3}$ \\ ${ }^{I}$ Faculty of Naval Architecture and Ocean Engineering, Istanbul Technical University, Maslak-Istanbul, Turkey \\ ${ }^{2}$ School of Marine Science and Technology, Newcastle University, Newcastle upon Tyne, UK \\ ${ }^{3}$ School of Naval Architecture and Ocean Engineering, Harbin Institute of Technology in Weihai, Shandong, China
}

\begin{abstract}
During the final quarter of the last century considerable efforts have been spent to reduce the hull pressure fluctuations caused by unsteady propeller cavitation. This has resulted in further changes in propeller design characteristics including increased skew, tip unloading and introduction of "New Blade Sections" (NBS) designed on the basis of the so-called Eppler code. An experimental study was carried out to investigate flow characteristics of alternative two-dimensional (2-D) blade sections of rectangular planform, one of which was the New Blade Section (NBS) developed in Newcastle University and other was based on the well-known National Advisory Committee for Aeronautics (NACA) section. The experiments comprised the cavitation observations and the measurements of the local velocity distribution around the blade sections by using a 2-D Laser Doppler Anemometry (LDA) system. Analysis of the cavitation tests demonstrated that the two blade sections presented very similar bucket shapes with virtually no width at the bottom but relatively favourable buckets arms at the suction and pressure sides for the NACA section. Similarly, pressure analysis of the sections displayed a slightly larger value for the NBS pressure peak. The comparative overall pressure distributions around the sections suggested that the NBS might be more susceptible to cavitation than the NACA section. This can be closely related to the fundamental shape of the NBS with very fine leading edge. Therefore a further investigation into the modification of the leading edge should be considered to improve the cavitation behaviour of the NBS.
\end{abstract}

KEY WORDS: New blade section; NACA; Cavitation behaviour; Velocity; Pressure distribution; Laser Doppler anemometry.

\section{NOMENCLATURE}

\begin{tabular}{|c|c|c|c|}
\hline$a$ & NACA mean line designation & $J_{\text {design }}$ & design propeller advance coefficient \\
\hline$A_{E} / A_{0}$ & expanded blade area ratio of & $P$ & pitch of propeller \\
\hline & propeller & $T I_{u}(\%)$ & axial component of the turbulence intensity \\
\hline$A R$ & aspect ratio of hydrofoil & & in percentage \\
\hline$b$ & span of hydrofoil & $t_{\max }$ & maximum thickness of hydrofoil \\
\hline$c$ & chord length of hydrofoil & $U_{m}$ & mean inflow speed of the tunnel based on the pitot \\
\hline$D$ & diameter of propeller & & measurements \\
\hline$C_{p}$ & non-dimensional pressure coefficient & $\bar{U}$ & mean velocity at the pickup point \\
\hline$f_{\max }$ & maximum camber of hydrofoil & $u$ & axial component of velocity \\
\hline
\end{tabular}

Corresponding author: Emin Korkut

e-mail:korkutem@itu.edu.tr 
fluctuation of $u$ component at the measuring point resultant velocity at the pickup point transverse component of velocity distance from leading edge of hydrofoil wall distance position

\section{Greek letters}

$\alpha \quad$ angle of attack of hydrofoil

$\delta_{\max } \quad$ maximum boundary layer thickness developed on hydrofoil obtained from Eq. (6)

$\lambda \quad$ wave length of laser beam

$\sigma_{i} \quad$ cavitation inception number

\section{INTRODUCTION}

When a hydrofoil or propeller blade section is designed, main emphases of the design are usually put on generating required lift with adequate structural strength and avoiding or reducing the effect of cavitation. On the other hand, propeller designers have traditionally used blade sections based on aerofoil profiles, which were developed for high lift coefficients, etc. However, marine propeller blade sections are usually designed with compromised lift coefficients for avoidance of cavitation or reduce it.

During the final quarter of the last century considerable efforts have been made to reduce the amplitudes of hull pressure fluctuations caused by unsteady propeller cavitation. This has resulted in changes in propeller design characteristics including increased skew, tip unloading and the introduction of "New Blade Sections" (NBS) designed on the basis of the so-called Eppler code (Eppler and Somers, 1980; Eppler and Shen, 1979). Eppler developed a method based on the conformal mapping technique for the design of a profile with a prescribed piece wise distribution of angles of attack around this profile. One of the main features of this method is to give a more accurate estimate of the velocity distribution in the leading edge region. Eppler and Shen (1979) applied this method to hydrofoil design. A profile called YS920 was developed to give a wider cavitation free bucket than those of the NACA mod.66 and NACA16-309 profiles.

Yamaguchi et al. (1988a; 1988b) addressed the problem by combining the Eppler code with a lifting surface design procedure. In their approach no attention was paid directly to the effect of blade section parameters, such as camber and thickness distributions, on hull surface pressure fluctuations. Lee et al. (1993) showed experimentally that the K18 section developed based on the new blade section displayed a wider cavitation free range than the existing NACA66 and NACA16 hydrofoil sections. The model propeller having this section experienced less cavitation extent and fluctuating pressure than the model propeller based on NACA section. This was also observed in the cavitation performances of the full-scale propellers (Lee et al., 1993).

Cavitation tunnel tests with 16 inches $(40.6 \mathrm{~cm})$ diameter model propeller with new blade section showed a 2 - 3 knots increase in cavitation inception speed, and reduced extent of cavitation, especially at high speeds, compared to the standard fleet DDG 51 propeller. Ship trials on the USS BARRY (DDG 52) with the new blade section propeller confirmed the improvements in propeller cavitation performance exhibited at model scale (Jessup et al., 1994). Dang et al. (1998) applied the new blade section approach to an aft foil section of a hydrofoil ferry and a highly skewed propeller. These profiles gave better cavitation performance than the traditional sections from NACA series. Kawakita and Hoshino (1998), and Zhou et al. (2001) showed that the propellers with new blade sections improved the open water characteristics and cavitation performances of the propeller with NACA sections. Dang (2004) also found that the new blade sections of a tanker propeller did have a wider cavitation bucket, reducing cavity volume on the suction side hence reducing pressure pulses. Li et al. (2010) used a new blade section to study for designing blade section in 3-D. Further review on the New Blade Section applications can be found in $19^{\text {th }}$ and $22^{\text {nd }}$ International Towing Tank Conferences, ITTC (1990; 1999).

Wang (1995) developed a propeller design method incorporating NBS design using both lifting surface theory and conformal mapping technique to achieve the required lift and cavitation characteristics on each individual blade section. The effect of maximum camber of a blade section on its cavitation and hydrodynamic characteristics was investigated and the so-called lift re-allocation was introduced to take into account of the wake variation in the circumferential direction. Then, pitch and maximum camber at each radius could be determined not only based on the lift, but also on the cavitation characteristics at that radius (Wang, 1995; Wang et al., 1995). Based on the methodology proposed in Wang (1995), a model propeller ECT102 was designed with the new blade sections. This propeller was a 5-bladed, right-handed, highly skewed propeller with a $0.24 \mathrm{~m}$ diameter, expanded blade area ratio of $A_{E} / A_{0}=0.726$ and pitch to diameter ratio of $P / D=0.918$. Another model propeller ECT103, which was a Meridian series with the same particulars, was also manufactured for the comparison. The ECT103 mo- 
del propeller had also highly-skewed blades which were represented by NACA sections. The main differences of two propellers were a slight variation in pitch and different thickness and camber distributions. Comparative cavitation tests with both propellers behind a dummy hull at the same operating condition $\left(J_{\text {design }}=0.608\right)$ indicated more or less similar cavitation patterns and similar magnitude of hull pressures. This was opposed to the theoretical predictions that indicated much wider cavitation buckets and reduced hull pressures for the NBS propeller (Wang, 1995). The reason for the above discrepancy could be differrent wake pattern from the one that the propellers were designed for. Therefore, an actual wake pattern of a containership was simulated in the cavitation tunnel, as shown in Fig. 1. Further tests behind the simulated wake also showed inconclusive results, indicating not appreciable improvement with the NBS propeller (Wang et al., 1995).

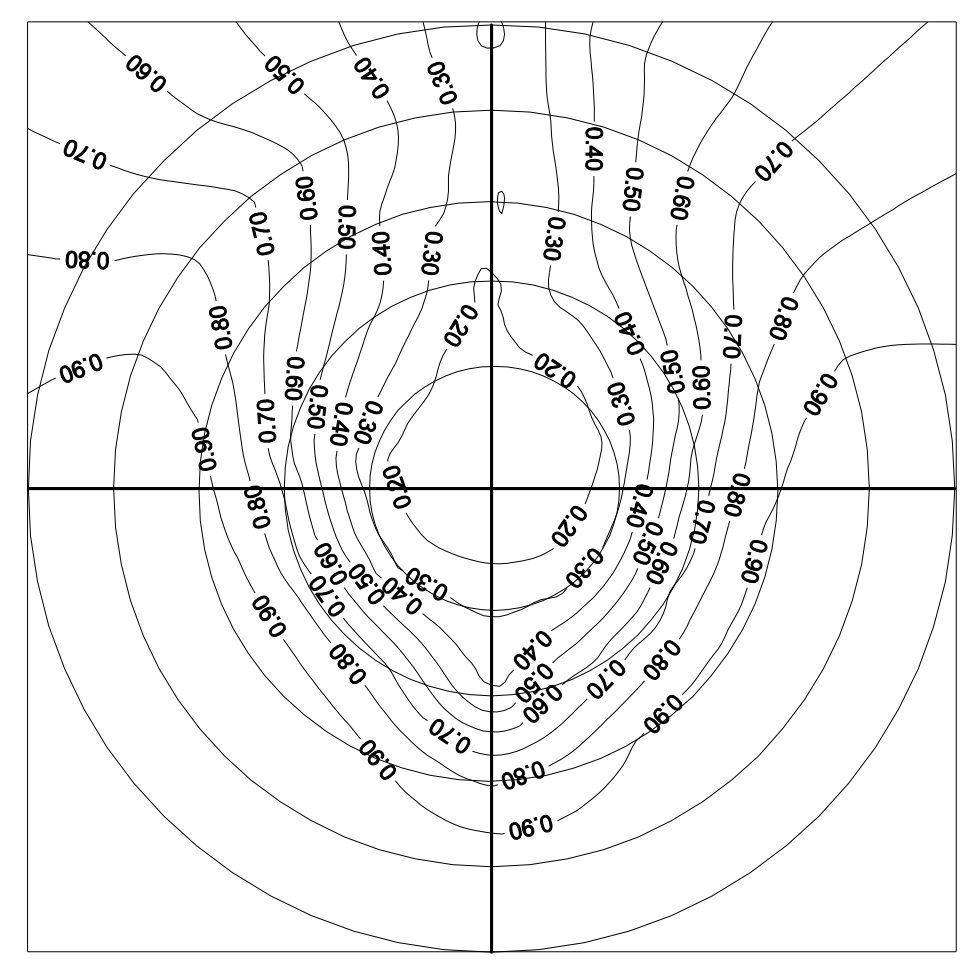

Fig. 1 Simulated wake pattern used in cavitation inception tests of ECT102 and ECT103 propellers.

The complexity of the theory and multitude of the parameters contributing to the discrepancy between the predictions and the measurements revealed that a further investigation into the sections of both NBS and NACA would be the most appropriate. This motivation was the basis for the experimental investigation presented in this paper.

\section{Objectives and scope of the study}

As was concluded in the previous section there was a need to study the cavitation bucket characteristics of two alternative blade sections, NBS and NACA, with the same chord, span length and maximum thickness but different distributions of the thickness and camber. Furthermore, it would be very informative to explore the bucket investigation by the measurements of the local velocity distribution around the representative blade sections using the Laser Doppler Anemometry (LDA) technique. This would provide information for likely peaks of the local velocities (pressures) on the blade sections and hence try to correlate these findings to the cavitation behaviours of the sections. Based on this information the shortcomings of the NBS section could be defined. The main objective of this study therefore was to understand why the NBS was failed for better cavitation performance.

Within the above context, two distinct sets of model tests were carried out with two rectangular planform of hydrofoils, one of which having the NBS other having a comparable NACA cross-section. The tests involved cavitation observations and detailed velocity measurements around the both sections by using a 2-D LDA system.

This paper presents the results of this investigation, in terms of the cavitation bucket and local velocity/pressure distribution 
around the blade sections in combination. The experimental set up and test conditions for the study are given in the following section. The results of the cavitation tests and the local velocity measurements around the blade sections are presented in the next. This is followed by the calculations of the pressure distributions around the sections and discussion for the comparison of the cavitation performances of the two sections by combining the results from the cavitation observations. Finally the main conclusions of the investigations are included.

\section{EXPERIMENTAL SET UP AND TEST CONDITIONS}

Experiments were carried out in two groups at the Emerson Cavitation Tunnel (ECT) of Newcastle University, which has a large rectangular test section with a cross-section of $1.22 \mathrm{~m}$ by $0.81 \mathrm{~m}(B x H)$. See Atlar (2000) for further details of the tunnel. The first group of the tests involved the cavitation inception measurements with two 2-D hydrofoils with alternative crosssections based on visual observations to establish their cavitation bucket diagrams. The second group comprised the measurements of local velocity around these sections at different angles of attack and at various distances from the surfaces of the hydrofoils.

In representing the typical blade sections of the earlier mentioned ECT propellers, two rectangular planforms with the specified blade sections were designed: one of which had a NACA66-004, $\mathrm{a}=0.8$ (modified) mean line blade section; other had a New Blade Section (NBS). Both hydrofoils had the same lengths of chord, span, maximum thicknesses and cambers but different distributions of the camber and thickness. Table 1 represents the main characteristics of the two blade sections (hydrofoils) while the comparative profiles of both sections are shown in Fig. 2. Table 2 displays the offset data for the NBS.

Table 1 Main particulars of test hydrofoils.

\begin{tabular}{|l|c|c|}
\hline \multicolumn{1}{|c|}{ Main Particulars } & NBS & NACA \\
\hline Chord length, $c,(\mathrm{~mm})$ & 250 & 250 \\
\hline Span, $b,(\mathrm{~mm})$ & 300 & 300 \\
\hline Aspect ratio, $A R$ & 1.2 & 1.2 \\
\hline Maximum thickness, $t_{\max },(\mathrm{mm})$ & 10 & 10 \\
\hline Maximum camber, $f_{\max },(\mathrm{mm})$ & 5 & 5 \\
\hline Location of maximum thickness $\%$ from the leading edge & 63 & 50 \\
\hline Location of maximum camber $\%$ from the leading edge & 63 & 50 \\
\hline Maximum thickness to chord length ratio, $t_{\max } / c$ & 0.04 & 0.04 \\
\hline Maximum camber to chord length ratio, $f_{\text {max }} / c$ & 0.02 & 0.02 \\
\hline
\end{tabular}

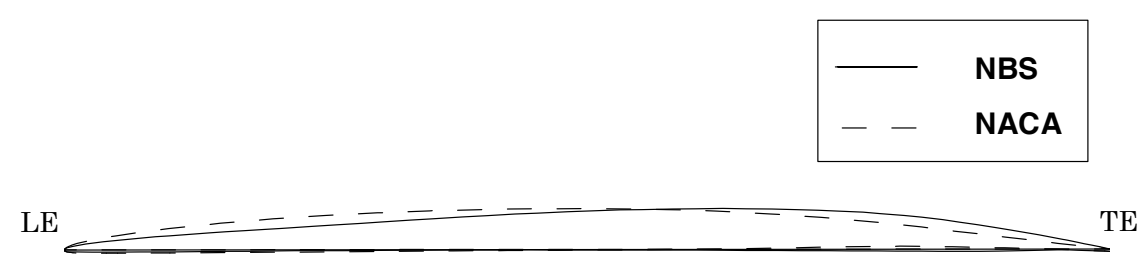

Fig. 2 Profiles of NBS and NACA hydrofoils.

Based on the simple beam theory and stressing procedure the hydrofoil stock was determined as $25.4 \mathrm{~mm}$ diameter for both hydrofoils. The hydrofoils were then manufactured from Nikalium alloy material using an NC machine with $\pm 0.005 \mathrm{~mm}$ tolerance. In the following sub-sections, the details of each group test are presented separately. 
Table 2 Offset data for NBS hydrofoil.

\begin{tabular}{|c|c|c|c|c|c|}
\hline $\mathrm{x} / \mathrm{c}$ & $\mathrm{y}_{\text {upper }}(\mathrm{mm})$ & $\mathrm{y}_{\text {lower }}(\mathrm{mm})$ & $\mathrm{x} / \mathrm{c}$ & $\mathrm{y}_{\text {upper }}(\mathrm{mm})$ & $\mathrm{y}_{\text {lower }}(\mathrm{mm})$ \\
\hline 0.000 & 0.000 & 0.000 & 0.480 & 9.178 & 0.050 \\
\hline 0.00016 & 0.145 & -0.105 & 0.520 & 9.590 & 0.036 \\
\hline 0.00032 & 0.205 & -0.177 & 0.560 & 9.896 & 0.000 \\
\hline 0.00064 & 0.295 & -0.250 & 0.600 & 10.072 & -0.012 \\
\hline 0.00096 & 0.365 & -0.305 & 0.640 & 10.095 & -0.032 \\
\hline 0.0016 & 0.470 & -0.370 & 0.680 & 9.942 & -0.090 \\
\hline 0.0024 & 0.565 & -0.420 & 0.720 & 9.595 & -0.135 \\
\hline 0.008 & 0.900 & -0.447 & 0.760 & 9.037 & -0.178 \\
\hline 0.040 & 2.050 & -0.510 & 0.800 & 8.250 & -0.225 \\
\hline 0.080 & 3.100 & -0.419 & 0.840 & 7.216 & -0.250 \\
\hline 0.120 & 3.869 & -0.380 & 0.880 & 5.917 & -0.250 \\
\hline 0.160 & 4.579 & -0.273 & 0.920 & 4.328 & -0.120 \\
\hline 0.200 & 5.207 & -0.210 & 0.956 & 2.624 & 0.040 \\
\hline 0.240 & 5.800 & -0.150 & 0.960 & 2.417 & 0.040 \\
\hline 0.280 & 6.386 & -0.081 & 0.968 & 1.993 & 0.000 \\
\hline 0.320 & 6.974 & -0.020 & 0.980 & 1.329 & -0.100 \\
\hline 0.360 & 7.563 & -0.010 & 0.992 & 0.750 & -0.220 \\
\hline 0.400 & 8.140 & 0.025 & 1.000 & 0.300 & -0.300 \\
\hline 0.4400 & 8.686 & 0.053 & & & \\
\hline
\end{tabular}

\section{Cavitation observations}

These tests provided a basis for establishing cavitation bucket diagrams of the test hydrofoils based on observations. Because of the physical limitations of the cavitation tunnel, the hydrofoils had to be hanged vertically from the top lid of the tunnel. This lid was equipped with a specially designed adjustable mechanism to hold the hydrofoil stock and provide the necessary angle of attack required to the hydrofoils. The vertical orientation coupled with relatively thicker cross-sections of the hydrofoils prevented to measure a clear inception for tip vortex cavitation. This was because the hydrofoil sections near the stock had a maximum thickness, which was nearly double the maximum thickness of the original cross-section, due to the strength requirements. This triggered early inception of cavitation at the top, and it was further complicated by the combined boundary layer of the lid and the hydrofoil. Therefore, under this circumstance, only the inception of the sheet cavitation at a suitable reference line could be recorded. This reference line was marked on the hydrofoils outside the fillet area at a distance of $140 \mathrm{~mm}$ from the top, as shown in Fig. 3, which was thought not affected by the top of the tunnel. Since the cavitation always developed from the top of the hydrofoils, the progress of the cavitation was allowed until the reference line.

Based on the above set-up the procedure for collecting an inception point for the sheet cavitation on the hydrofoils was processed as follows. Initially the tunnel static pressure was set to a constant value and the tunnel water velocity was increased until the extent of sheet cavitation reached and passed the reference line by a reasonable amount. The tunnel speed was then decreased until the extent of sheet cavitation was steadily just reaching at the reference line. Based on a number of repetitive observations the corresponding point was, then, accepted as the valid inception of the sheet cavitation, which was the desinent point. In order to obtain a reasonable bucket, angle of attack was varied between $-1^{\circ}$ and $4^{\circ}$ 


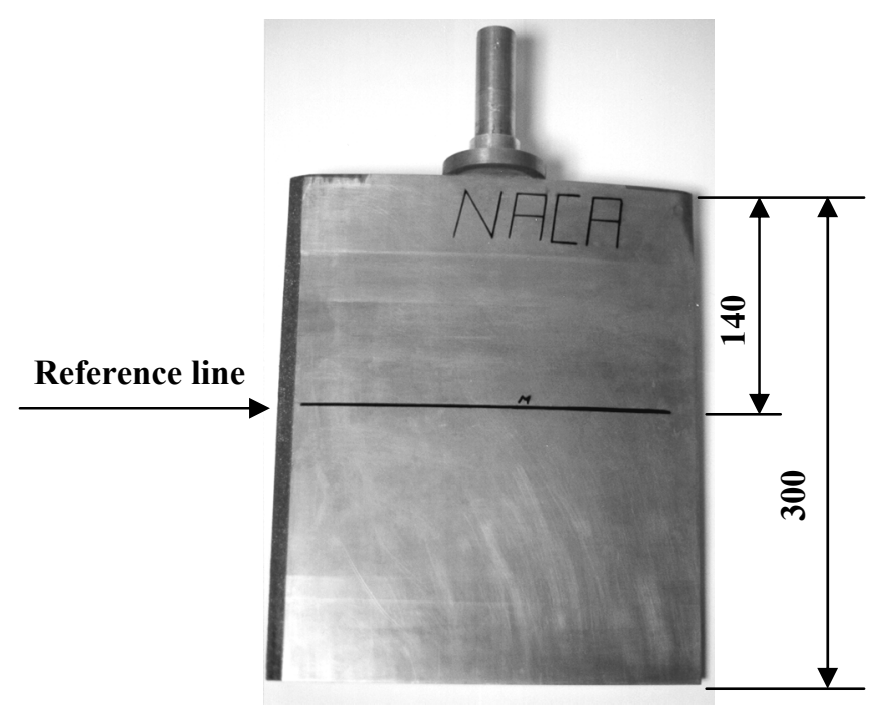

Fig. 3 A general view of the one of the rectangular planform hydrofoils showing the marked reference line.

During these tests, the dissolved gas content level was measured by using a Jenway DO2 meter 9071 apparatus and kept at about $35 \%$ while the chord based Reynolds number varied between $1.3 \times 10^{6}$ and $1.75 \times 10^{6}$. The results of the cavitation inception measurements of the hydrofoils are shown in Fig. 5 and related discussions are presented in the corresponding section.

The uncertainty analysis used in the tests followed that of the American National Standard Institute (ANSI) and American Society of Mechanical Engineers (ASME) standard on measurement uncertainty, which is based on the root-sum square (RSS) method (ASME, 1998). Total uncertainty in a measurement is the combination uncertainty due to systematic error and uncertainty due to random error. Uncertainty, $\mathrm{U}$, is defined as an interval, about the measured value, that has a pre-assigned probability of containing true value. Therefore the interval $\bar{X} \pm U$ represents a band about the measurement mean, $\bar{X}$, within which the true value is expected with respect to a given level of confidence (ASME, 1998). Uncertainty levels of the cavitation inception tests were $\pm 3.65 \%$ and $\pm 5.25 \%$ for the $95 \%$ and $100 \%$ confidence levels, respectively. Fig. 4 shows the angle of attack setting in the measurements and uncertainty for the angle of attack setting was $\pm 0.1^{\circ}$.

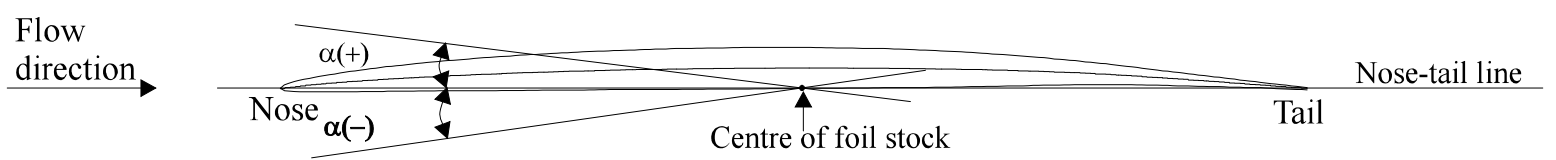

Fig. 4 Angle of attack setting during the measurements.

\section{Local velocity measurements by LDA}

For the measurements of the local velocities around the hydrofoils, a 2-D Laser Doppler Anemometry (LDA) system of DANTEC was used. The system had a Spectra Physics 2016 type, water-cooled laser with a power of 5 watt. The details of the system are given in Atlar et al. (1997).

Four laser beams, two in the axial direction and two in the transverse, were generated from a $600 \mathrm{~mm}$ diameter of optic probe in a backward scatter system and driven by a two-dimensional traverse mechanism. The LDA system consisted of a laser light source, a beam separator, fiber-optic couplers, a multi-color receiver, a signal processor, a personal computer, a fiber-optic probe, and a two-axis computer-driven traverse with a resolution of $10 \mu \mathrm{m}$. The laser beam was separated into green $(\lambda=514.5$ $\mathrm{nm})$ and blue $(\lambda=488 \mathrm{~nm})$ lights. The optical elements included a $40 \mathrm{MHz}$ Bragg cell to remove directional ambiguity and a $600 \mathrm{~mm}$ focal length lens. Therefore the beam diameters of the green and blue lights were $2.18 \mathrm{~mm}$ and $2.08 \mathrm{~mm}$, respectively while sizes of the measuring volume of the LDA system were $2.294 \times 10^{-2} \mathrm{~m}^{3}$ and $2.176 \times 10^{-2} \mathrm{~m}^{3}$ for the green and blue lights, respectively. 
The traversing system driving the probe was placed on a bench underneath the tunnel and the test hydrofoils. The laser beams approached the reference line (shown in Fig. 3) around the hydrofoils through the bottom observation window with an angle of $3.66^{\circ}$ with respect to the vertical plane through the hydrofoil section. This was necessary to approach to the hydrofoil surface without being obstructed by the bottom part of the hydrofoil. Of course this small inclination would have a small effect on the velocity components which was later corrected based on the simple cosine relationship.

The trajectory of the traversing system was adjusted separately for the suction and pressure side of the hydrofoils and the velocity data for each point along the each side of the hydrofoils was collected accordingly. It was decided that the local velocity measurements should be carried out at least 2 different tunnel flow speeds, 5 different angles of attack and 5 different distances from the surface (wall) of the hydrofoils to obtain as much as information for the detailed flow characteristics. In order to get a fine distribution around the hydrofoils, particularly at the leading edge region, a reasonable number of pickup points were used. A summary of the test conditions are displayed in Table 3.

Table 3 Test conditions for cavitation tests based on observation and local velocity measurements.

\begin{tabular}{|l|c|c|}
\hline \multicolumn{1}{|c|}{ Parameters tested } & Cavitation tests & Local velocity measurements \\
\hline Hydrofoil section type & NBS and NACA & NBS and NACA \\
\hline Inflow speed, $U_{m},(\mathrm{~m} / \mathrm{s})$ & 4.9 to 7 & 3 and 6 \\
\hline Angle of attack, $\alpha$ & $-1^{\circ}$ to $4^{\circ}$, & $-3^{\circ}, 0^{\circ}, 3^{\circ}, 6^{\circ}, 9^{\circ}$ \\
\hline Distance from the wall, $y_{b},(\mathrm{~mm})$ & N/A & $0.25,0.5,1,2$ and 6 \\
\hline Number of velocity pickup points at suction side & N/A & 31 and 29 for NBS and NACA, respectively \\
\hline Number of velocity pickup points at face & N/A & 29 and 31 for NBS and NACA, respectively \\
\hline Cavitation number based on inflow speed, $\sigma_{v}$ & same & same \\
\hline Dissolved gas content level & about $35 \%$ & about $35 \%$ \\
\hline
\end{tabular}

Before each test the particular hydrofoil was fixed at the desired angle of attack. The traversing system was calibrated for its start and finish positions fixed at the nose and tail respectively by using the measuring volume of the laser. Between the nose and tail it was made sure that the measuring volume would be following the reference line on the hydrofoil.

Following the above process the traversing mechanism driven by the DANTEC BURSTware software operated the probe to collect data at previously specified velocity pickup points. For each pick up point 15 seconds was spent and data (approximately 3000 samples) were collected at a rate varying between 50 and $6000 \mathrm{~Hz}$, depending on the pickup points. The Doppler signals read at each data point for the axial and transverse velocity components were processed by DANTEC Burst Spectrum Analyzer, BSA, which was set and controlled by the BURSTware software. The uncertainty levels for the $u$ (axial) and $v$ (transverse) components of the velocity, including the position of measuring point setting, were $\pm 2.6 \%$ and $\pm 4.7 \%$, respectively for $95 \%$ confidence level.

\section{PRESENTATION AND DISCUSSION OF TESTS RESULTS}

\section{Cavitation observations}

As shown in Fig. 5, there is a clear trend that both sections present similar bucket diagrams but the NACA section displays a relatively favourable cavitation bucket compared to the NBS, particularly at the suction side of the hydrofoil. In practical terms, at relatively large positive angles of attack the NBS will experience a sheet cavitation at the suction side about a half degree earlier than the NACA section to experience the same cavitation extent. This advantageous margin is approximately halved for the pressure side cavitation of the NACA hydrofoil, at relatively small positive and negative angles of attack.

As noticed in the bucket diagrams, there is no width at the bottom of the buckets for both hydrofoils. In spite of the observation difficulties number of repetitive points were taken at this min $\sigma_{i}$ region (i.e., small angle of attack and very high velocities), and it was observed that this trend was persistent. 


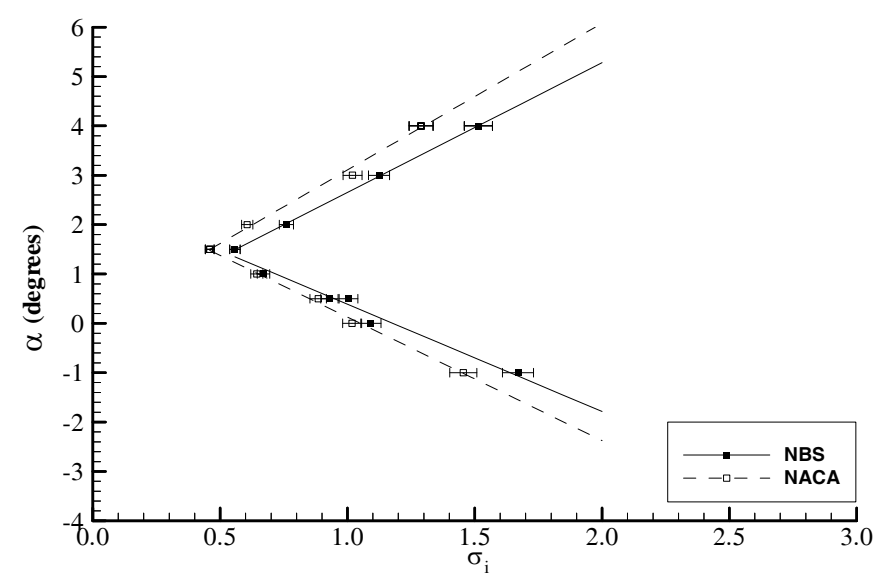

Fig. 5 Comparison of cavitation bucket diagram of NBS hydrofoil with that of NACA hydrofoil.

During the tests the onset of the cavitation was always observed at the leading edge of the two sections. At negative angle of attacks to positive angle of attacks of $1^{\circ}$ a "pressure side" cavitation was observed while at angle of attacks greater than $1^{\circ} \mathrm{a}$ "suction side" cavitation was observed. The nature of the cavitation onset at the face was very sharp and sudden appearing as a "streak" type along the span of the hydrofoil sections. However, the onset for the suction side cavitation was gradually starting from the top of the hydrofoil like a wedge shape and developing as a "sheet" cavitation.

One may suggest that the set-up of the cavitation tests did not lend itself to investigate the tip vortex type cavitation due to the physical limitations of the hydrofoil attachment, hence only the sheet type was investigated. Although the shape of cavitation bucket diagrams is not a typical bucket type, these diagrams would still give useful information about comparison of relative cavitation behaviours of these hydrofoils.

\section{Local velocity measurements by LDA and calculated pressure distributions}

The local velocity components in the axial and transverse directions were collected by BURSTware software (Atlar et al., 1997) and stored in appropriate files. The axial component was in-line with the direction of the tunnel incoming flow, while the transverse component was horizontal to the axial component. From these files it was possible to obtain mean velocities, turbulence intensities as well as the time history of the collected data using this software.

In the following the mean velocity components $u$ in the axial direction and $v$ in the transverse direction were used for the presentation of the velocity distributions and the calculation of the pressures around the hydrofoils. The mean velocities $u$ and $v$ are non-dimensionalised by the mean inflow speed, $U_{m}$, of the tunnel based on the pitot measurements as:

$$
\frac{u}{U_{m}} \text { and } \frac{v}{U_{m}}
$$

Distance from the leading edge of the hydrofoil, $x$, is non-dimensionalised by the chord length as $\frac{x}{c}$ and $x / c=0.0$ represents the leading edge while $x / c=1.0$ represents the trailing edge. The wall distance values, $y_{\mathrm{b}}$, are non-dimensionalised by the maximum boundary layer thickness developed on hydrofoil, $\delta_{\max }$, obtained from Eq. (6) as $\frac{y_{b}}{\delta_{\max }}$.

In representing the pressure distribution around the hydrofoils, the pressure values are calculated by means of the Bernoulli equation and expressed in terms of non-dimensional pressure coefficient, $C_{p}$, as:

$$
C_{p}=1-\left(\frac{u_{r}}{U_{m}}\right)^{2}
$$

where $u_{r}$ is the resultant velocity at the pickup point given by $u_{r}=\left(u^{2}+v^{2}\right)^{1 / 2}$. 
Cavitation bucket diagrams are also produced from local velocity measurements such as

$$
\sigma_{i}=-C_{p \min }
$$

The main objective of this group of the tests was to investigate the fundamental characteristics of the velocity, and hence pressure distribution around the NBS hydrofoil relative to its counterpart NACA hydrofoil, which represents a typical propeller blade section. The combined analysis of the local velocity measurements and earlier conducted cavitation observations with these hydrofoils could provide an answer why the earlier performed cavitation and hull pressure tests with the two model propellers, one of which had the NBS type section and the other had the NACA type section, presented inconclusive results such that no improvement has been achieved in the cavitation and hence hull pressure performance in contrast to the favourable predictions from the theory.

During the local velocity measurements, a wide range of test parameters was selected such that 2 hydrofoil types, 2 inflow speeds, 5 angles of attack, 5 distances from the wall and 60 velocity pickup points around the suction and pressure side of the hydrofoils. Although a detailed analysis of these effects could be very useful as a separate investigation in the long term, the present discussion is focused on the effect of the hydrofoil types on the velocity and hence pressure distribution, which is directly related to the cavitation characteristics.

As far as the angle of attack is concerned, the selected range of $-3^{\circ}, 0^{\circ}, 3^{\circ}, 6^{\circ}$ and $9^{\circ}$ was wider than the range selected in the bucket analysis obtained from the cavitation tests based on observation, which varied between $-1^{\circ}$ and $4^{\circ}$. However it was thought that such a wide range should reflect the trend in more dramatic fashion.

Detailed flow measurements (i.e. local velocity measurements) were performed at five different values of distances from the hydrofoil surfaces varying $0.25 \mathrm{~mm}$ to $6 \mathrm{~mm}$. Local Reynolds number is defined as

$$
R_{e x}=U_{m} x / v
$$

where $U_{m}$ is the mean inflow flow velocity, $x$ is the distance from the leading edge and $v$ is the kinematic viscosity of water.

During the tests the maximum Reynolds number value for $3 \mathrm{~ms}^{-1}$ was $8.88 \times 10^{5}$. As the critical Reynolds number for a flat plate is given by Schlichting and Gersten $(2000)$ as $1 \times 10^{6}$, the boundary layer developed on the hydrofoils could be accepted as in the laminar and then in transitional flow regime for $3 \mathrm{~ms}^{-1}$. Therefore the boundary-layer thicknesses for a smooth flat plate at a distance $x$ from the leading edge and at zero incidence for laminar and turbulent flows were given by Schlichting and Gersten (2000) as:

$$
\delta=5 \sqrt{\frac{v x}{U_{m}}} \quad \text { for laminar flow }
$$

and

$$
\delta=0.37 x R_{e x}^{-0.2} \quad \text { for turbulent flow }
$$

Both formulae may be used to calculate the local boundary layer thicknesses along the hydrofoils in an approximate manner. The local boundary layer thicknesses obtained from the formulae were in the range of 0 to $1.33 \mathrm{~mm}$ for the laminar flow and 0 to $6 \mathrm{~mm}$ for the turbulent flow. One would expect that the maximum boundary layer developed on the hydrofoils would be less than $6 \mathrm{~mm}$ due to the pressure gradient. Furthermore the leading edge is the stagnation point and the boundary layer thickness increases as we move away from the leading edge along the chord. Therefore the data acquired at the $x / c$ positions around the leading edge may be accepted as outside the boundary layer developed for at least one or two wall distances, which is an interest of the study for cavitation. 
Background free-stream turbulence survey of the cavitation tunnel was carried by using the same 2-D LDA system and the axial component of the turbulence intensity, $T I_{u}$ was defined as:

$$
T I_{u}(\%)=\frac{\sqrt{\overline{u^{\prime 2}}}}{\bar{U}} 100
$$

where $u^{\prime}$ is the fluctuation of $u$ component at the measuring point and $\bar{U}$ is the mean velocity at that point. The survey results indicated that the axial component $(u)$ of the background turbulence intensity of the tunnel test section was about $2 \%$ at $U_{m}=3$ $m s^{-1}$ (Atlar and Korkut, 1997).

Fig. 6 shows the axial component of turbulence intensity distribution on the suction side of the NBS hydrofoil at $\alpha=3^{\circ}$ and $U_{m}=3 \mathrm{~ms}^{-1}$ for different angles of attack. As the tunnel has background turbulence intensity about $2(\%)$, the average turbulence intensity measured for the hydrofoil around the leading edge region is also about 2 (\%) (Fig. 6). This shows that the free-stream flow during the measurements had inherent turbulence level similar to the background turbulence level of the tunnel. Turbulence levels increase mainly as the $x / c$ position increases and with increased angles of attack the increase in turbulence is also experienced.

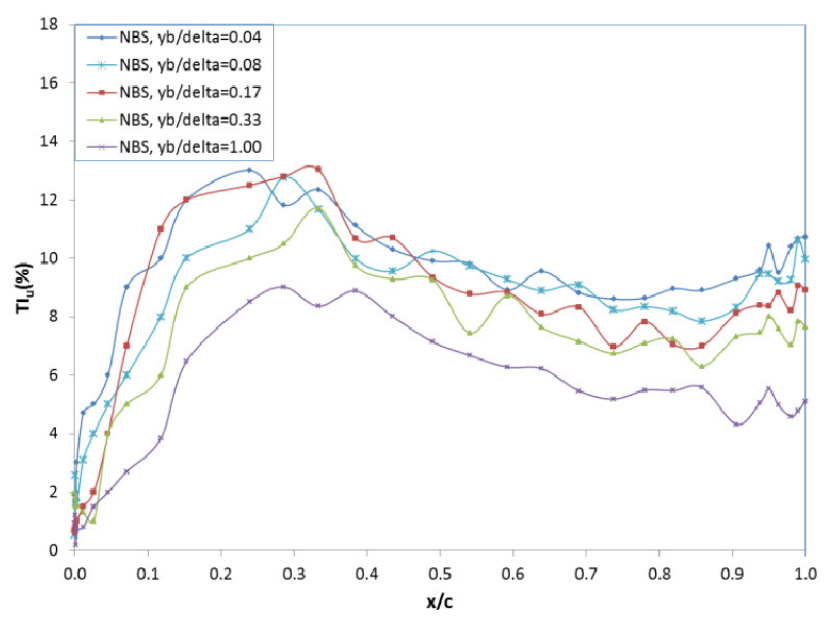

(a) $\alpha=-3^{\circ}$.

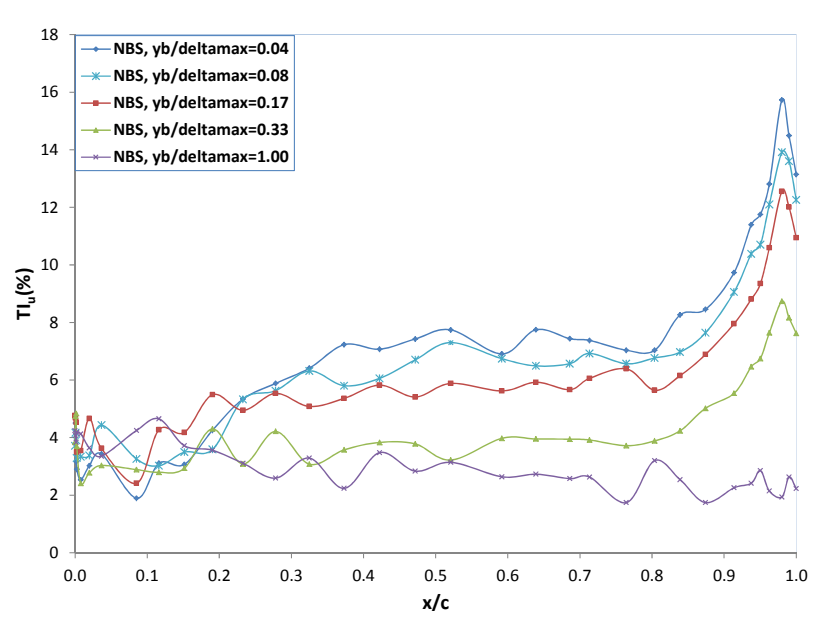

(c) $\alpha=3^{\circ}$.

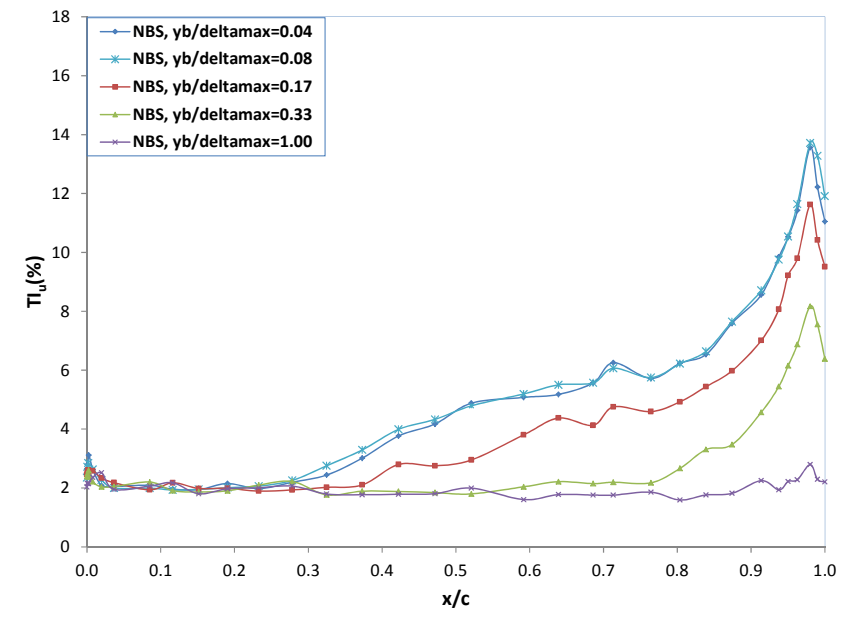

(b) $\alpha=0^{\circ}$.

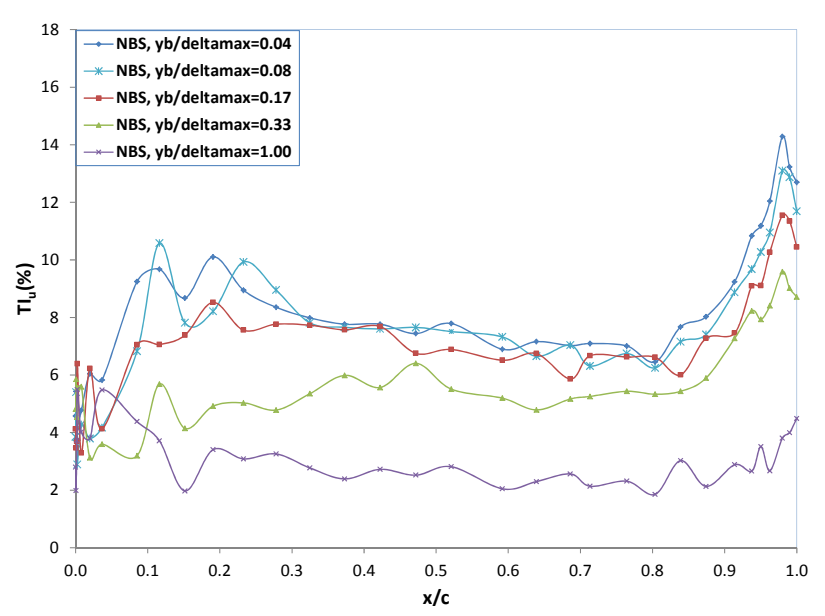

(d) $\alpha=6^{\circ}$.

Fig. 6 Axial component of turbulence intensity $\left(T I_{u}(\%)\right)$ distribution on the suction side of NBS hydrofoil at $U_{m}=3 \mathrm{~ms}^{-1}$ : (a) $\alpha=-3^{\circ}$; (b) $\alpha=0^{\circ}$; (c) $\alpha=3^{\circ}$; (d) $\alpha=6^{\circ}$. 
Although the local velocity measurements carried out at 5 angles of attack, two tunnel inflow velocities and 5 wall distances, here the results are presented only for $y_{b} / \delta_{\max }=0.04,0.17 \& 1.00$ at $\alpha=-3^{\circ}$ and $U_{m}=3 \mathrm{~ms}^{-1}$, as shown in Fig. 7 to display a general trend. As shown in this Fig. the transverse component of the local velocity, $v$, is generally small compared to the magnitude of the axial component of the local velocity, $u$. The transverse component reaches to its maximum values, which would be as large as $30 \%$ of the axial component, only around the nose and tail regions. Therefore the resultant local velocity will be dominated by the axial component.

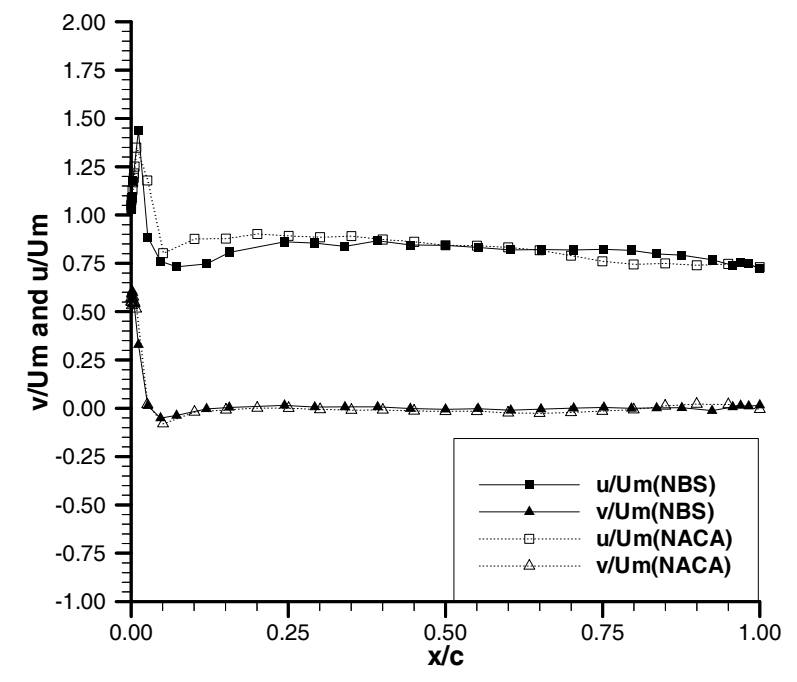

(a) $y_{b} / \delta_{\max }=0.04$

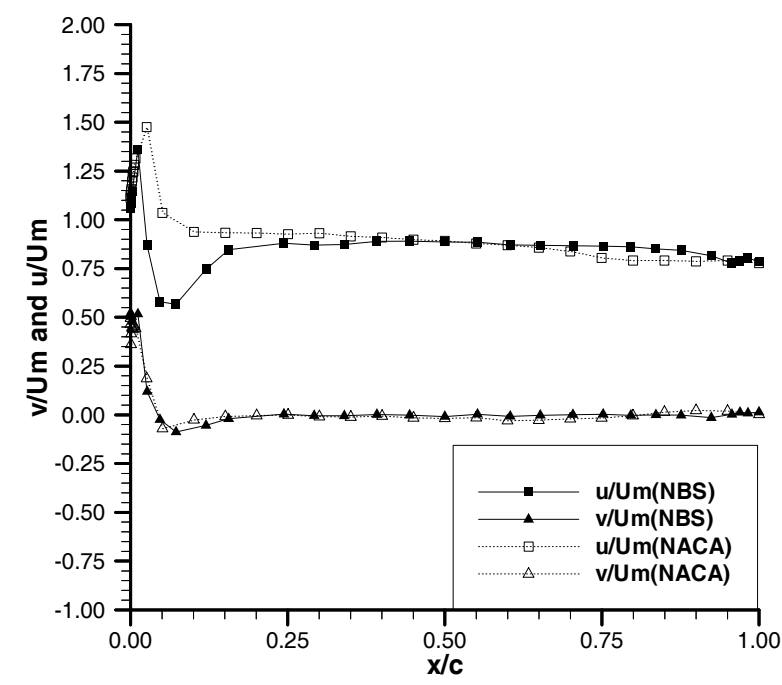

(b) $y_{b} / \delta_{\max }=0.17$.

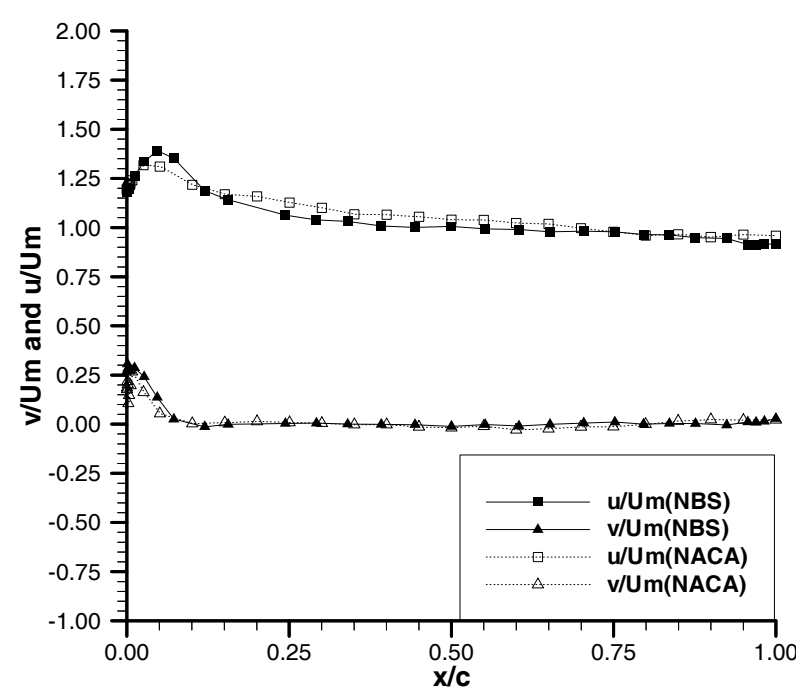

(c) $y_{b} / \delta_{\max }=1.00$.

Fig. 7 Comparison of local velocity distributions on the pressure side of NBS hydrofoil with NACA hydrofoil at $\alpha=-3^{\circ}$ and $U_{\mathrm{m}}=m s^{-1}$ : (a) $y_{b} / \delta_{\max }=0.04$; (b) $y_{b} / \delta_{\max }=0.17$; (c) $y_{b} / \delta_{\max }=1.00$.

In Figs. 8 through 12, the comparison of the pressure distribution around the NACA and NBS hydrofoils are presented for each angle of attack at various measuring distances from the wall for the inflow velocity of $3 \mathrm{~ms}^{-1}$. The pressure coefficient, $C_{p}$ values were determined using the Bernoulli equation. Although the use of this equation including the boundary layer effect may cast doubt about the cavitation inception predictions at the leading edge, the minimal effect of the boundary layer in this region may justify its use for cavitation comparisons. 
For angles of attack of $-3^{\circ}$ and $0^{\circ}$, both hydrofoils clearly indicate a strong tendency for sharp suction peaks at the face of the hydrofoils and hence a mechanism to generate a face cavitation. As the angle of attack is increased to positive $3^{\circ}, 6^{\circ}$ and $9^{\circ}$ the suction peaks are observed at the suction side of both hydrofoils and hence displaying a mechanism for the suction side cavitation. For all the angles of attack tested, the suction peaks appear around the leading edge of both hydrofoils which is less than $3-4 \%$ of the chord length from the nose.

The magnitudes of the suction peak of the two hydrofoils at the leading edge are comparable. Although there are differences in the peaks from one case to another, these differences are not considerable and do not present any trend. However there is a very clear trend in the build-up of the suction peak for each hydrofoil such that the NACA hydrofoil displays larger suction compared to the NBS over the forward half of the hydrofoil while the NBS displays larger suction in comparison to the NACA over the aft half of the hydrofoil. Therefore the pressure gradient of NBS at the leading edge is much higher compared to the NACA section.

In order to investigate the above trend, one should consider the comparative pressure distribution of the two hydrofoils for each angle of attack. As shown in Fig. 8 at the angle of attack of $-3^{\circ}$ the pressure side suction peaks of the two hydrofoils at the

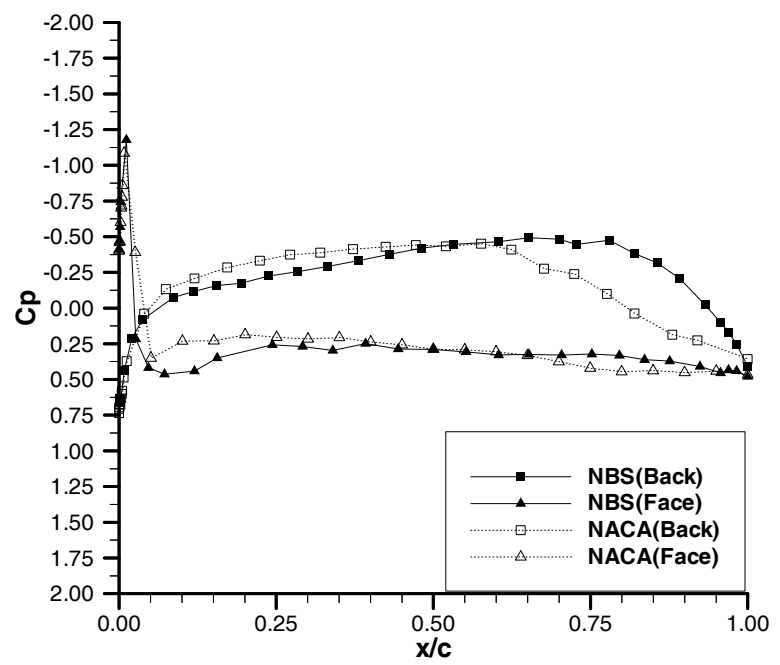

(a) $y_{b} / \delta_{\max }=0.04$.

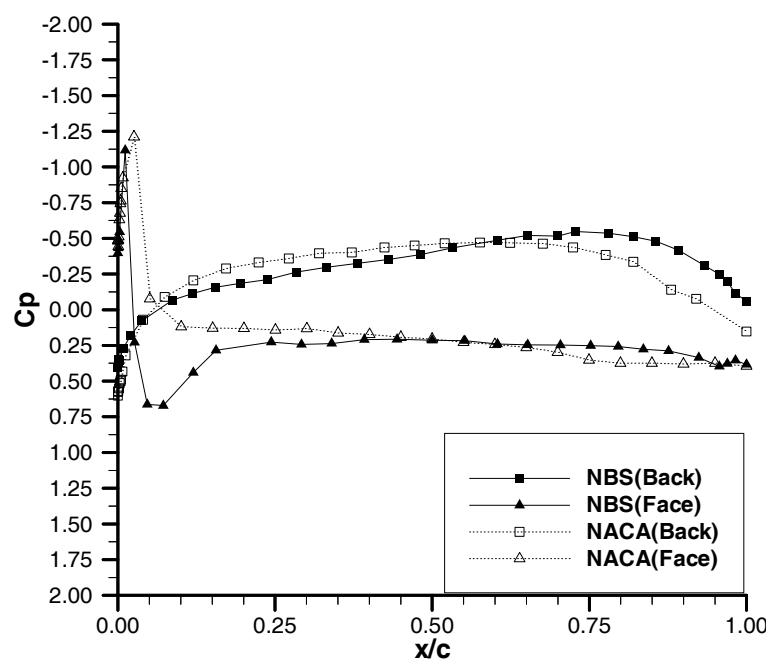

(b) $y_{b} / \delta_{\max }=0.17$.

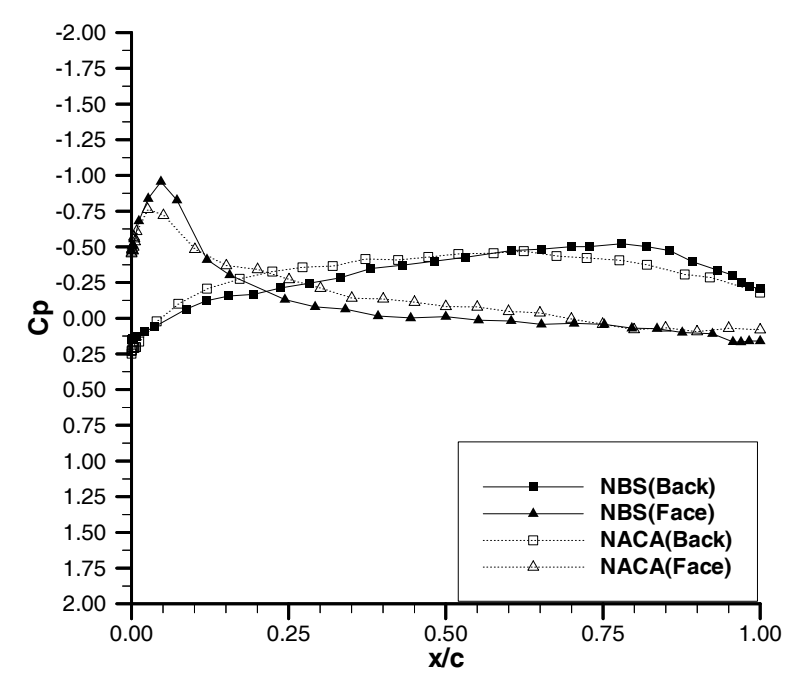

(c) $y_{b} / \delta_{\max }=1.00$.

Fig. 8 Comparison of local pressure distributions of NBS hydrofoil with NACA hydrofoil at $\alpha=-3^{\circ}$ and $U_{m}=3 \mathrm{~ms}^{-1}$ : (a) $y_{b} / \delta_{\max }=0.04$; (b) $y_{b} / \delta_{\max }=0.17$; (c) $y_{b} / \delta_{\max }=1.00$. 
leading edge are comparable for varying wall distances displaying similar trends. Over the majority of the forward half of the hydrofoil, the tendency to produce more suction at the pressure and suction sides of the hydrofoil is more prominent for the NACA compared to the NBS. However at the leading edge NBS displays remarkably strong suction peak which is comparable or even worse in some cases.

If one considers the pressure distribution at angle of attack $0^{\circ}$, as shown in Fig. 9, although the magnitude of the suction and its gradient is reduced, the previous trend can be still observed. When the angle of attack is set to $3^{\circ}$, as shown in Fig. 10 , the suction peak is generated at the suction side of the both hydrofoils, as expected, but the earlier observed trend in terms of the pressure distribution and the large pressure gradient at the leading edge of the NBS hydrofoil is still obvious.

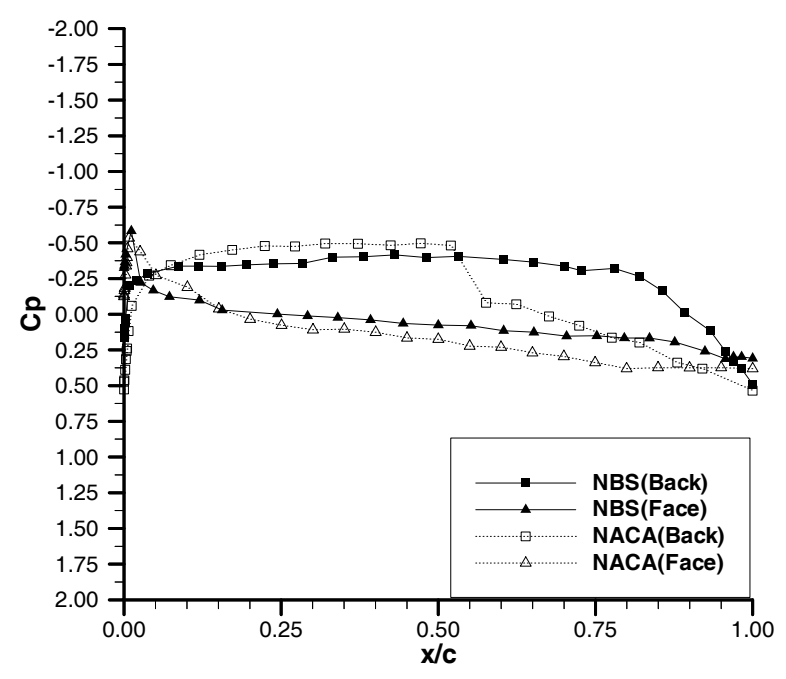

(a) $y_{b} / \delta_{\max }=0.04$.

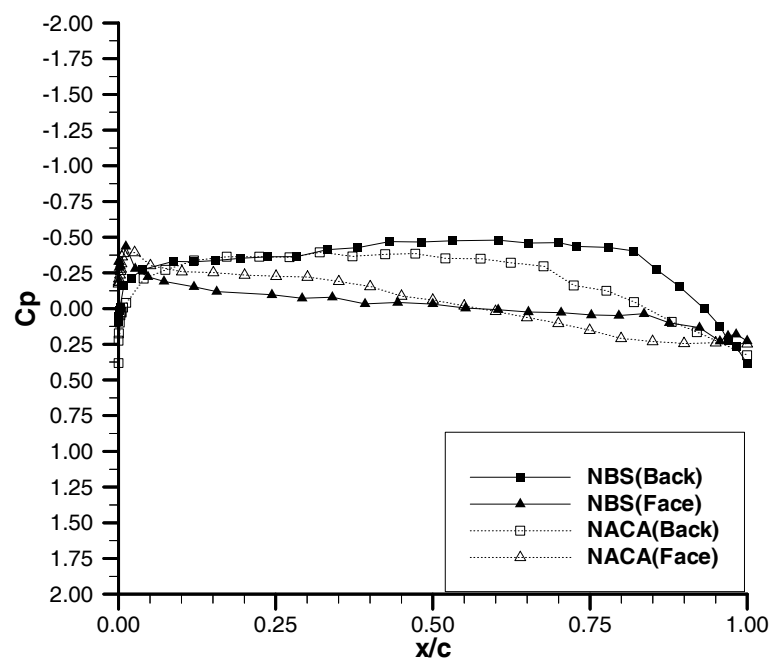

(b) $y_{b} / \delta_{\max }=0.17$.

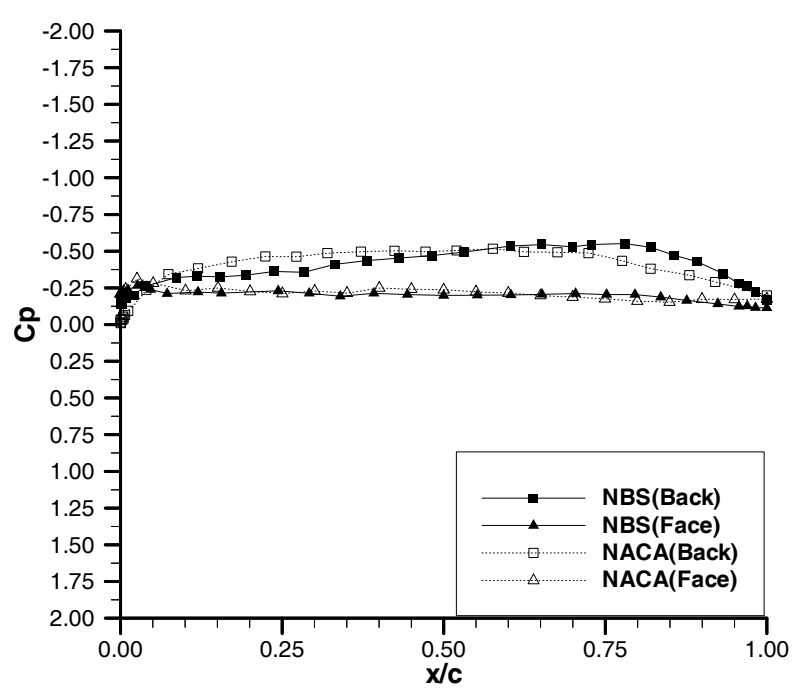

(c) $y_{b} / \delta_{\max }=1.00$.

Fig. 9 Comparison of local pressure distributions of NBS hydrofoil with NACA hydrofoil at $\alpha=0^{\circ}$ and $U_{m}=3 \mathrm{~ms}^{-1}$ : (a) $y_{b} / \delta_{\max }=0.04$; (b) $y_{b} / \delta_{\max }=0.17$; (c) $y_{b} / \delta_{\max }=1.00$.

In Fig. 11, the comparative pressure distribution is given at an angle of attack of $6^{\circ}$. The trend for the pressure distribution at the suction side is still the same whilst at the pressure side both hydrofoils display similar distribution. Fig. 12 shows the pressure distribution at an angle of attack of $9^{\circ}$. In spite of the oscillations in the pressure distribution the earlier trend can be observed. At this angle of attack strong flow separation was observed during the tests. 


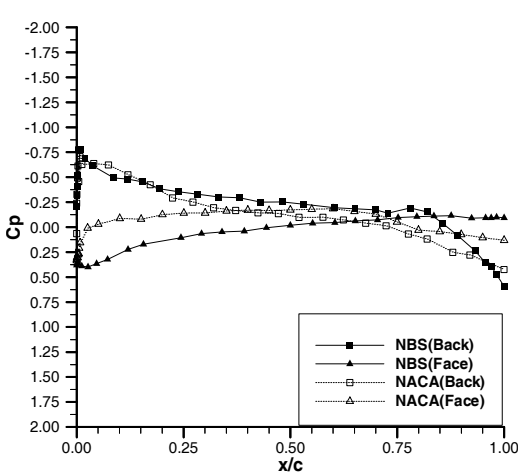

(a) $y_{b} / \delta_{\max }=0.04$.

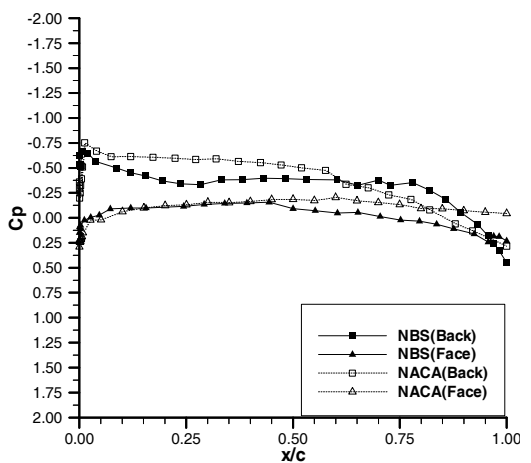

(b) $y_{b} / \delta_{\max }=0.17$.

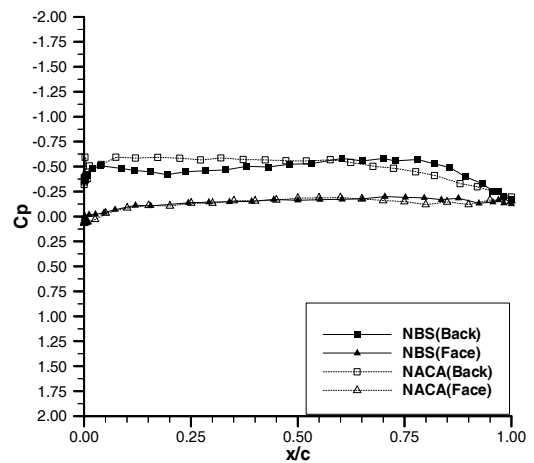

(c) $y_{b} / \delta_{\max }=1.00$.

Fig. 10 Comparison of local pressure distributions of NBS hydrofoil with NACA hydrofoil at $\alpha=3^{\circ}$ and $U_{m}=3 \mathrm{~ms}^{-1}$ : (a) $y_{b} / \delta_{\max }=0.04$; (b) $y_{b} / \delta_{\max }=0.17$; (c) $y_{b} / \delta_{\max }=1.00$.

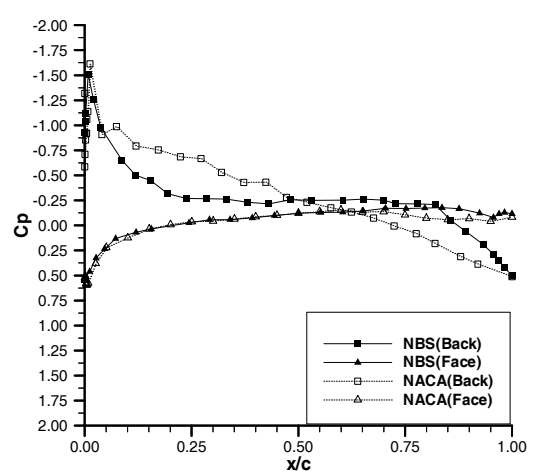

(a) $y_{b} / \delta_{\max }=0.04$.

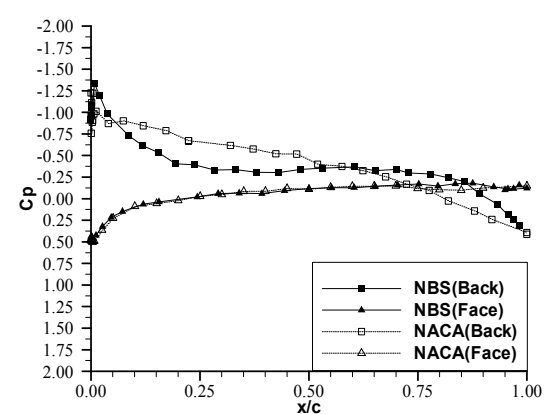

(b) $y_{b} / \delta_{\max }=0.17$.

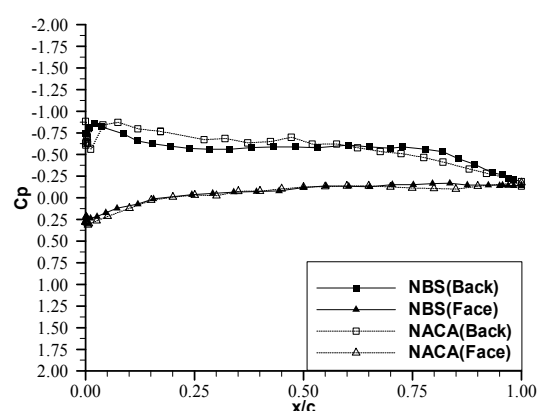

(c) $y_{b} / \delta_{\max }=1.00$.

Fig. 11 Comparison of local pressure distributions of NBS hydrofoil with NACA hydrofoil at $\alpha=6^{\circ}$ and $U_{m}=3 \mathrm{~ms}^{-1}$ : (a) $y_{b} / \delta_{\max }=0.04$; (b) $y_{b} / \delta_{\max }=0.17$; (c) $y_{b} / \delta_{\max }=1.00$.

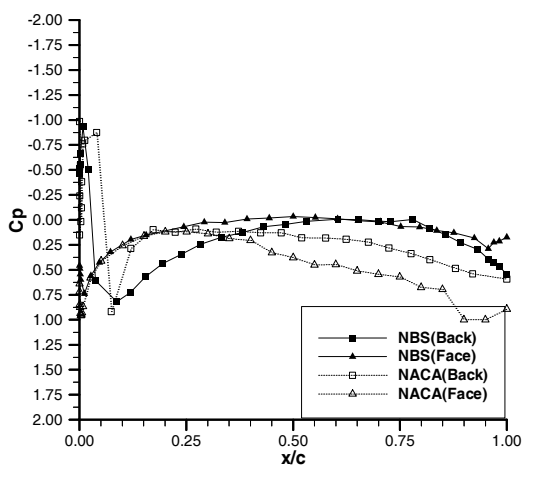

(a) $y_{b} / \delta_{\max }=0.04$.

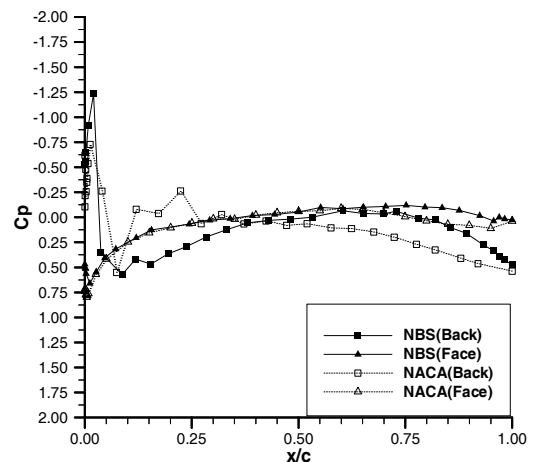

(b) $y_{b} / \delta_{\max }=0.17$.

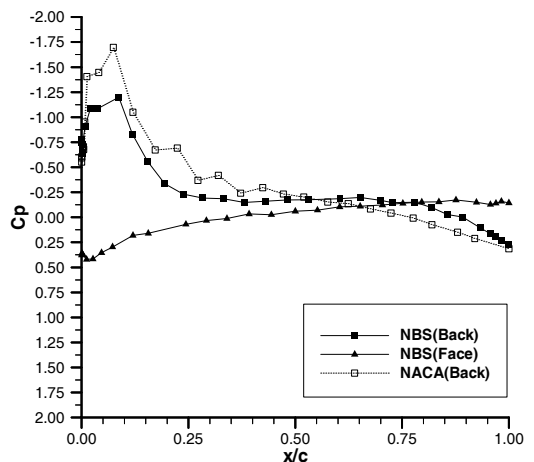

(c) $y_{b} / \delta_{\max }=1.00$.

Fig. 12 Comparison of local pressure distributions of NBS hydrofoil with NACA hydrofoil at $\alpha=9^{\circ}$ and $U_{m}=3 \mathrm{~ms}^{-1}$ : (a) $y_{b} / \delta_{\max }=0.04$; (b) $y_{b} / \delta_{\max }=0.17$; (c) $y_{b} / \delta_{\max }=1.00$.

It is obvious that the above observed trend in the pressure distribution, which displays strong tendency to generate suction at the forward half of the NACA hydrofoil and relatively less but similar tendency to generate suction at the aft half of the NBS hydrofoil, can be directly related to the position of the maximum thickness for each hydrofoil. The NBS section with its maximum thickness being located behind the mid-chord displays weaker suction effect at the forward half of the hydrofoil compared to the NACA section whose maximum thickness is located at the mid-chord of the hydrofoil and hence provides strong suction effect. 
Figs. 13 and 14 show comparisons of the local pressure distributions on the pressure/suction side of NBS and NACA hydrofoils for different wall distances at $U_{m}=3 \mathrm{~ms}^{-1}$, respectively. As the wall distance increases minimum pressure value around the leading edge also reduces, indicating the effect of viscosity in the boundary layer. As expected the minimum pressure value reduces if the angle of attack is increased.

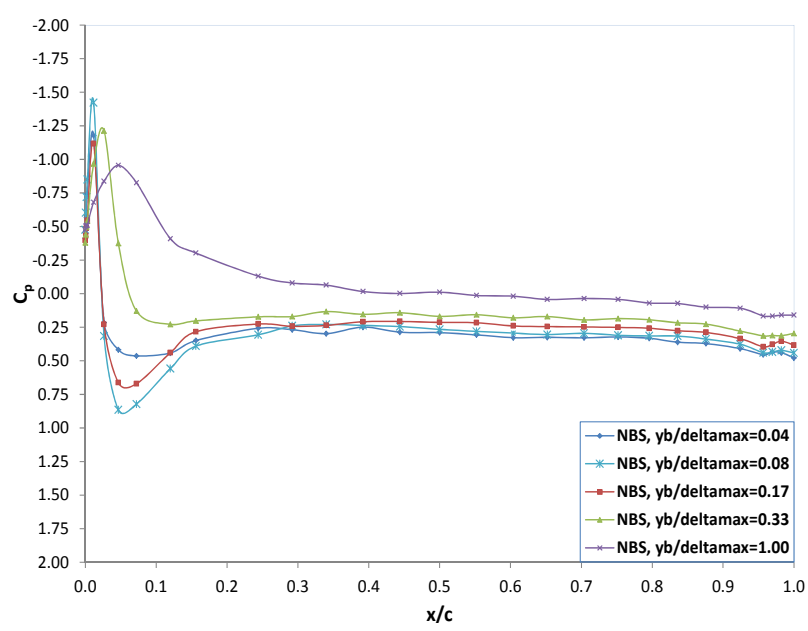

(a) $\alpha=-3^{\circ}$ (pressure side).

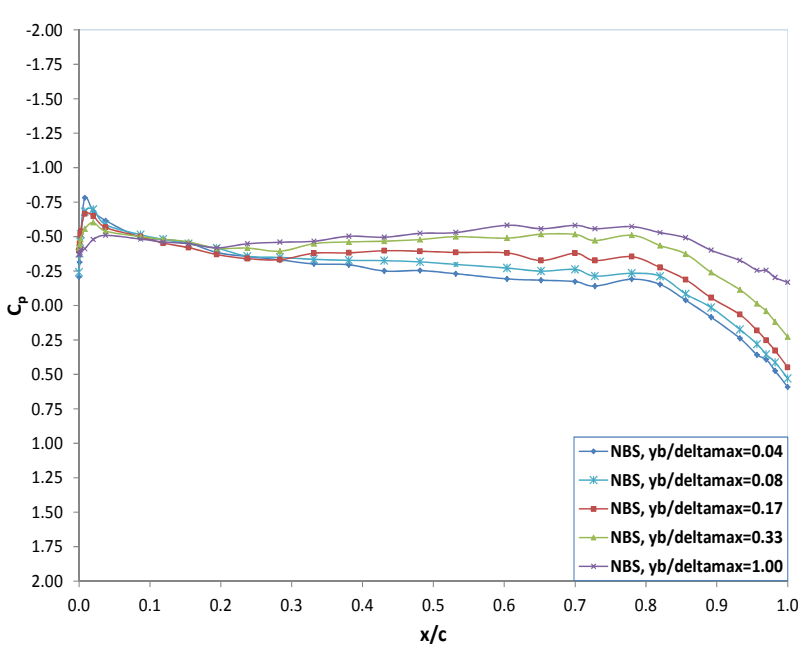

(c) $\alpha=3^{\circ}$ (suction side).

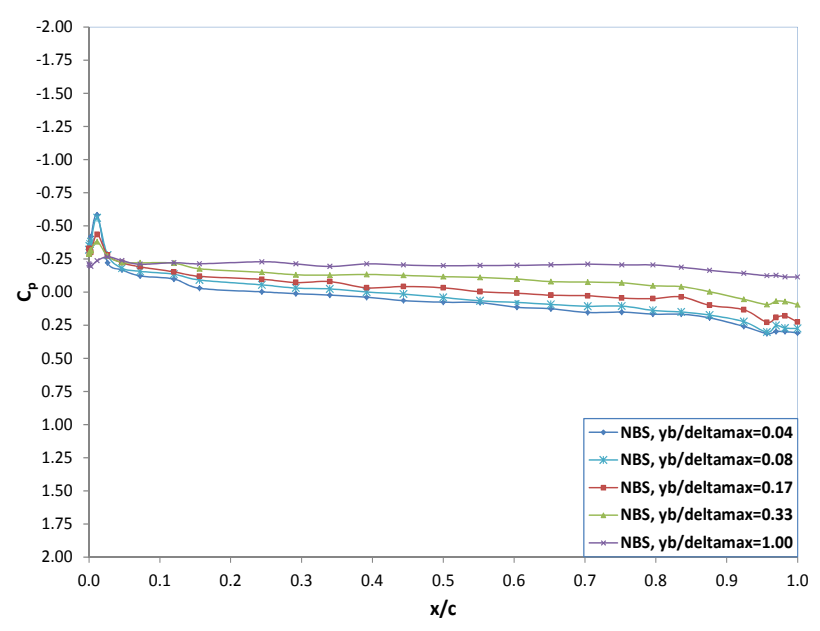

(b) $\alpha=0^{\circ}$ (pressure side).

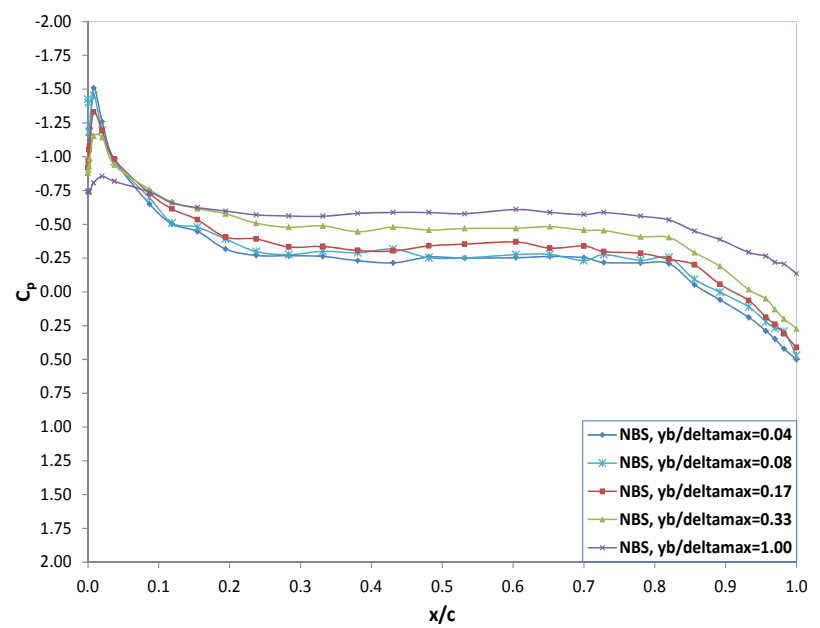

(d) $\alpha=6^{\circ}$ (suction side).

Fig. 13 Comparison of local pressure distributions on the pressure/suction side of NBS hydrofoil for different wall distances at $U_{m}=3 \mathrm{~ms}^{-1}$ : (a) $\alpha=-3^{\circ}$ (pressure side); (b) $\alpha=0^{\circ}$ (pressure side); (c) $\alpha=3^{\circ}$ (suction side); (d) $\alpha=6^{\circ}$ (suction side).

Fig. 15 shows cavitation bucket diagrams of both hydrofoils produced from the LDA measurements. In this figure angle of attack of 9 degrees is not included due to the flow separation and vortices formed at that angle which caused a backward flow and pressure drop close to section surfaces of both hydrofoils. As shown in Fig. 15 the NBS hydrofoil displays a narrower cavitation bucket than NACA hydrofoil between angles attack of -3 to 3 degrees except at the non-dimensional wall distances of 0.17 and 0.33 . This trend is generally consistent with the cavitation observation tests although the types of cavitation are differrent, streak or sheet type for the cavitation tests based on observation and tip vortex type based on the LDA measurements. Beyond the angle attack of 3 degrees it is difficult to draw a conclusion. 


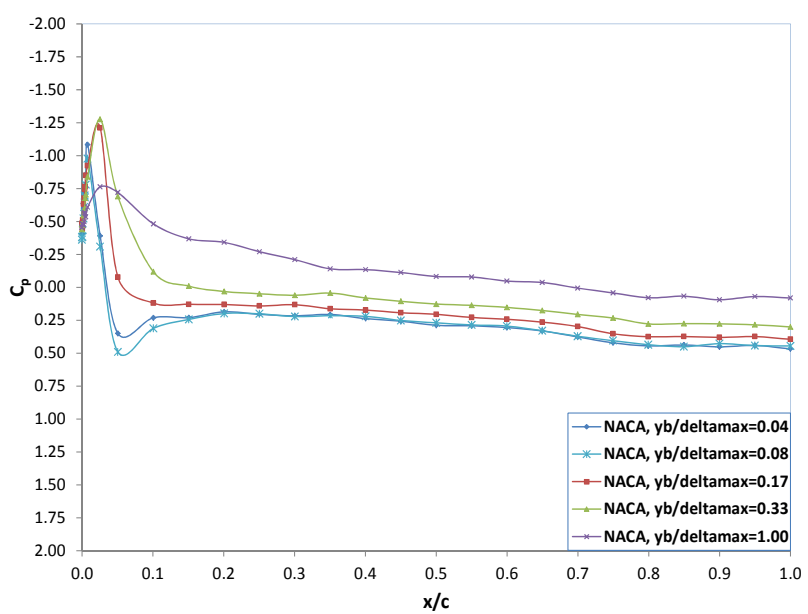

(a) $\alpha=-3^{\circ}$ (pressure side).

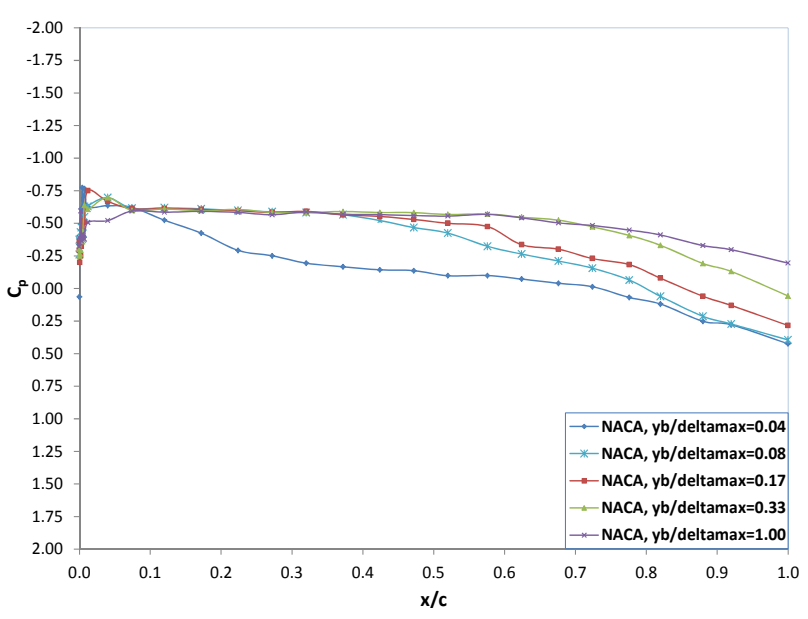

(c) $\alpha=3^{\circ}$ (suction side).

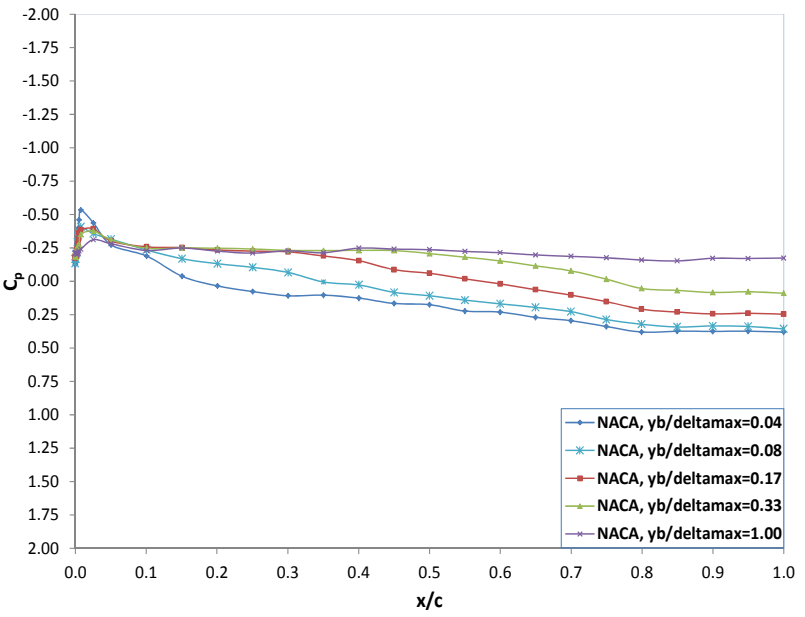

(b) $\alpha=0^{\circ}$ (pressure side).

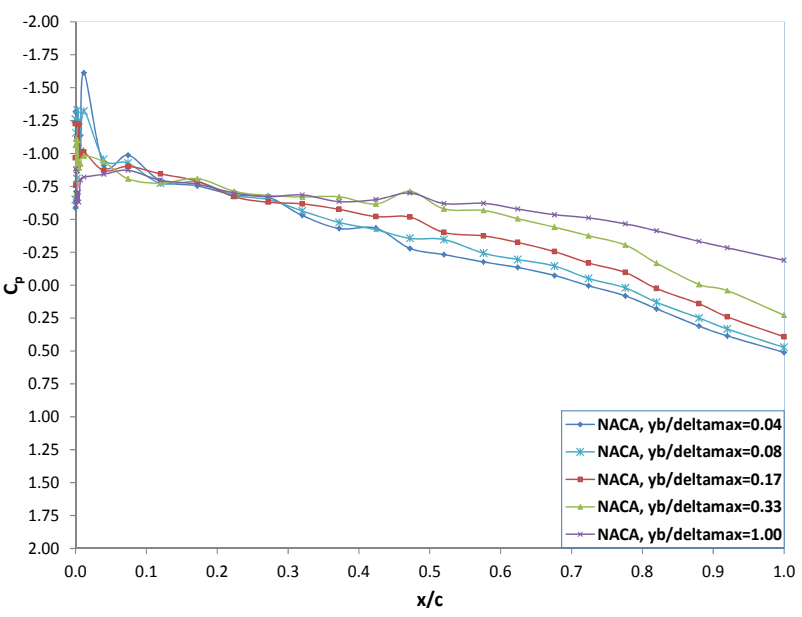

(d) $\alpha=6^{\circ}$ (suction side).

Fig. 14 Comparison of local pressure distributions on the pressure/suction side of NACA hydrofoil for different wall distances at $U_{m}=3 \mathrm{~ms}^{-1}$ : (a) $\alpha=-3^{\circ}$ (pressure side);

(b) $\alpha=0^{\circ}$ (pressure side); (c) $\alpha=3^{\circ}$ (suction side); (d) $\alpha=6^{\circ}$ (suction side).

Bearing in mind the earlier stated considerations, if one attempts to relate the findings from the pressure analysis to the findings from the bucket analysis based on the observations, there is consistency between two test results which can be described as follows:

Although the range of the angle of attack tested in the velocity measurements (i.e. $-3^{\circ}$ to $9^{\circ}$ ) is much larger than the bucket tests (i.e. $-1^{\circ}$ to $4^{\circ}$ ), the cavitation onset observed in the inception tests was always at the leading edge for both hydrofoils regardless it was suction side or pressure side cavitation. This observation is well supported by the trend in the pressure distribution.

During the inception tests, as the angle of attack was ranged from $-1^{\circ}$ to a small positive value of the angle of attack, the observed cavitation was the pressure side cavitation and its onset was sudden as a flash at the leading edge. At relatively large positive angles of attack up to a maximum of $4^{\circ}$ the observed cavitation was suction side cavitation and its onset was gradual contrary to the sudden pressure side cavitation. This observation is also well supported by the pressure distribution analysis such that at $-3^{\circ}$ and $0^{\circ}$ of angles of attack the leading edge pressure peak develops on the pressure side of the hydrofoils while at $3^{\circ}$, $6^{\circ}$ and $9^{\circ}$ the pressure peaks are at the suction side. The gradient of the pressure at the pressure side of the hydrofoils for $-3^{\circ}$ and $0^{\circ}$ is much higher than the gradient of the pressure at the suction side of the hydrofoil for $3^{\circ}$ of comparable positive angle of attack. Therefore the sudden appearance of the pressure side cavitation in comparison to the gradual built-up of the suction side cavitation can be related to the high pressure gradient at the negative and $0^{\circ}$ values of angle of attack. 


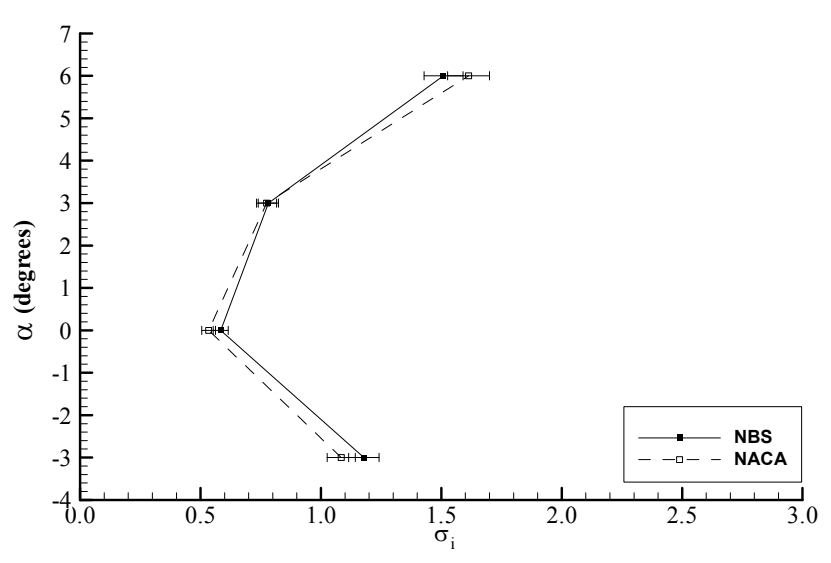

(a) $\mathrm{y}_{\mathrm{b}} / \delta_{\max }=0.04$.

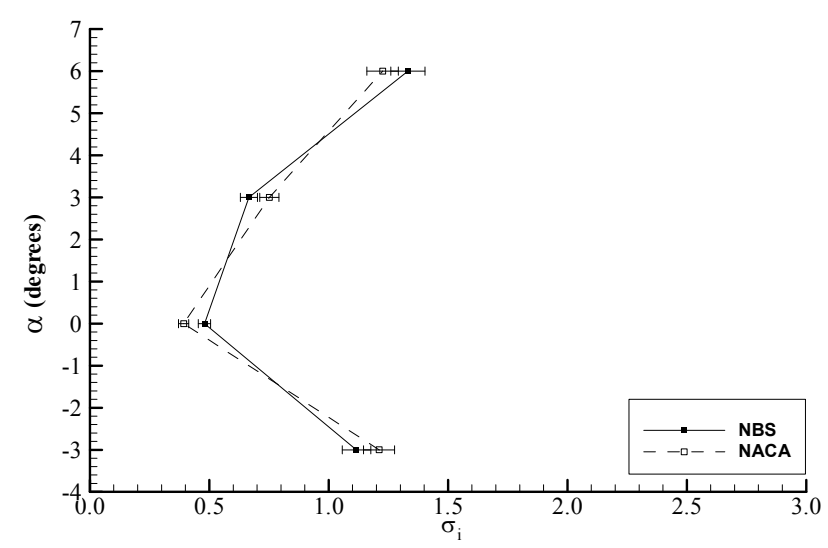

(c) $\mathrm{y}_{\mathrm{b}} / \delta_{\max }=0.17$.

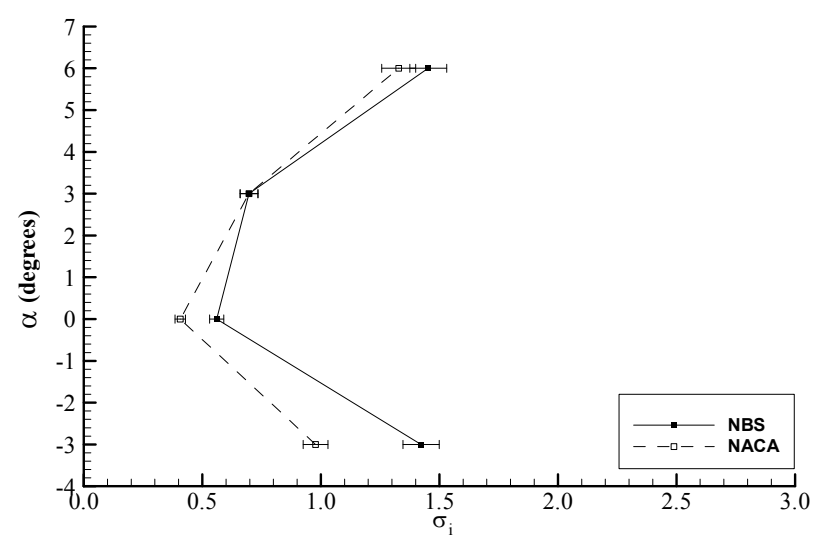

(b) $\mathrm{y}_{\mathrm{b}} / \delta_{\max }=0.08$.

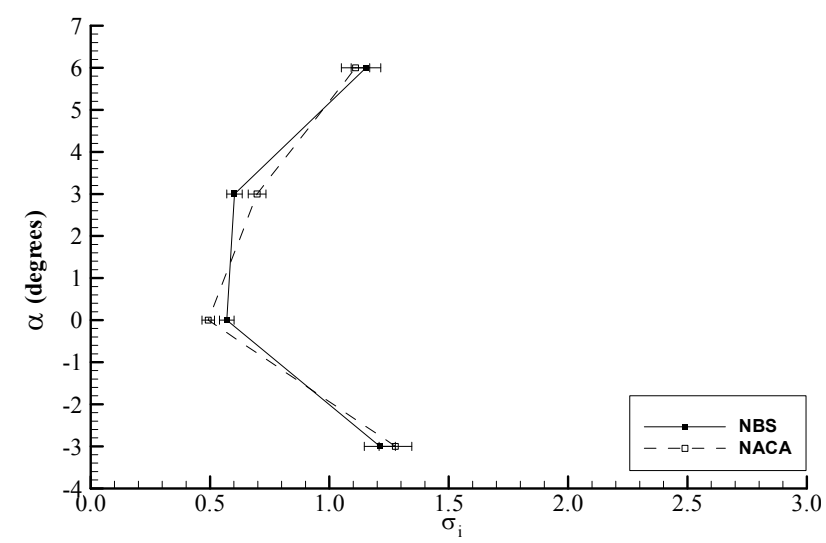

(d) $\mathrm{y}_{\mathrm{b}} / \delta_{\max }=0.33$.

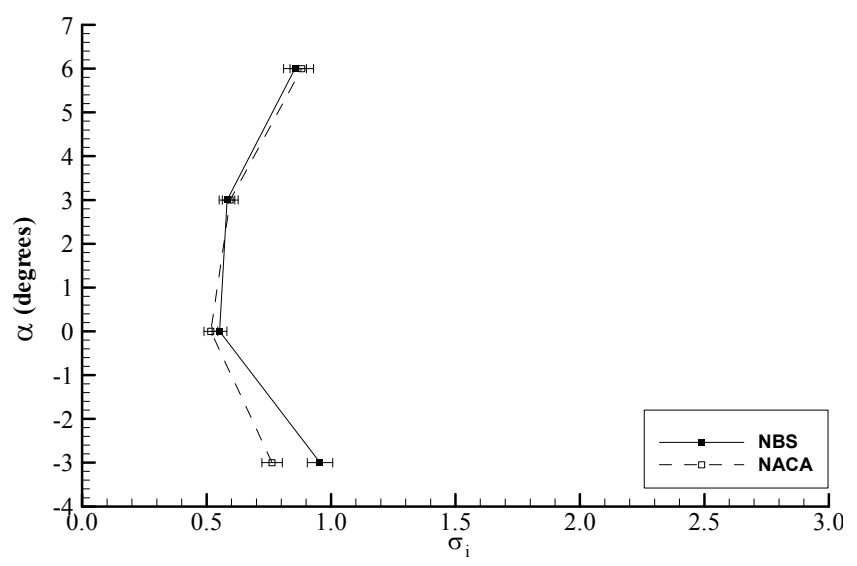

(e) $\mathrm{y}_{\mathrm{b}} / \delta_{\max }=1.00$.

Fig. 15 Comparison of cavitation bucket diagram of NBS hydrofoil with NACA hydrofoil obtained from LDA measurements at $\mathrm{U}_{\mathrm{m}}=3 \mathrm{~m} / \mathrm{s}$ : (a) $\mathrm{y}_{\mathrm{b}} / \delta_{\max }=0.04$; (b) $\mathrm{y}_{\mathrm{b}} / \delta_{\max }=0.08$; (c) $\mathrm{y}_{\mathrm{b}} / \delta_{\max }=0.17$; (d) $\mathrm{y}_{\mathrm{b}} / \delta_{\max }=0.33$; (e) $\mathrm{y}_{\mathrm{b}} / \delta_{\max }=1.00$.

Based upon the above discussion one can conclude that the trend in the cavitation buckets of the two hydrofoils obtained from the laser measurements supports the trend in the cavitation buckets of the hydrofoils. Nevertheless, the comparative analysis of the buckets indicated a relatively small but favourable bucket for the NACA compared to the NBS between angles of attack of -3 and 3 degrees. The trend in the pressure distribution of the NBS hydrofoil indicates that this section behaves like a flat plate at an angle of attack which generates large suction peak and high pressure gradient because of its flat and sharp entry 
section at the forward due to the location of the maximum thickness shifted behind the mid-chord. In fact this analogy for the pressure distribution between the NBS section and a flat plate can be seen in Fig. 16 based on the pressure measurements taken over a flat plate for various angles of incidence in a wind tunnel by Crompton and Barrett (2000). It is clear that the minimum pressure develops at the leading edge and this is followed by a region of strong pressure recovery (i.e. large pressure gradient) before settling to a zero pressure gradient further downstream. In the same study it is also interesting to note that, as shown in Fig. 17, the stagnation point hardly moves until the angle of incidence for the flat plate reaches at about $20 \mathrm{deg}$. The significance of this observation in relation to the NBS is that while one can expect the location of the stagnation point for a typical foil section with a round shape leading edge will change depending upon the angle of attack, this will be severely delayed for a flat plate and hence similar behaviour for the NBS. Furthermore there will be no significant boundary layer developed from the leading edge region that will further justify the use of the Bernoulli equation in some confidence around the leading edge.

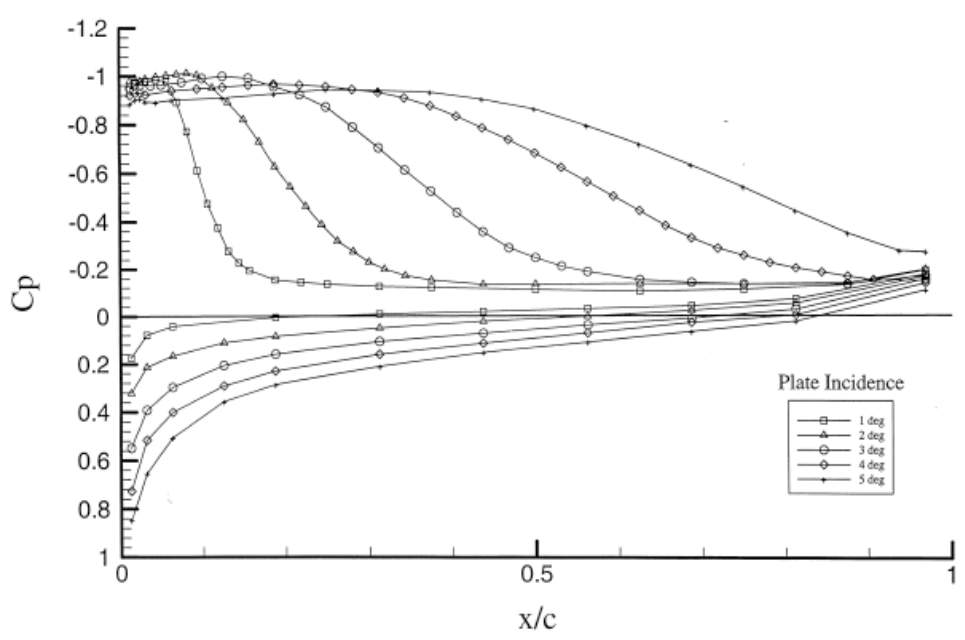

Fig. 16 Pressure coefficient distribution around a flat plate for various angles of incidence $\left(\mathrm{Re}_{\mathrm{c}}=2.13 \times 10^{5}\right)$, Crompton and Barrett $(2000)$.

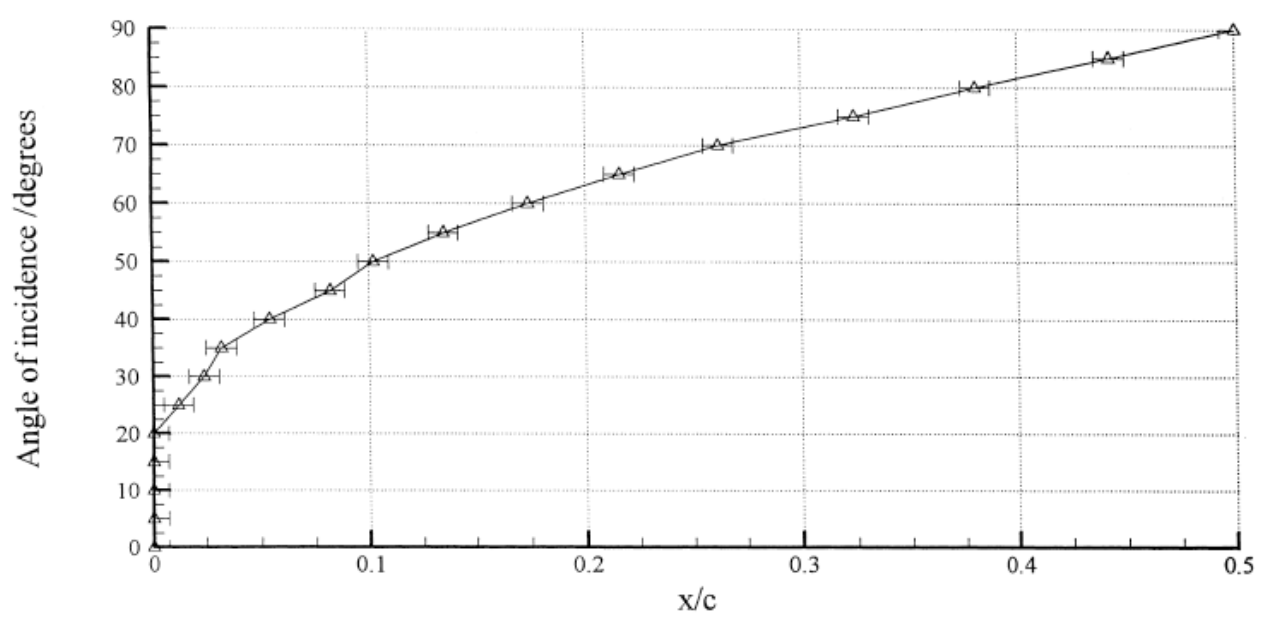

Fig. 17 Location of the forward stagnation point for a flat plate for various angles of incidence $\left(\mathrm{Re}_{\mathrm{c}}=0.2 \times 10^{5}\right)$, Crompton and Barrett $(2000)$.

Because of this fact the leading edge cavitation characteristics of the NBS profile are relatively worse than the NACA profile. In essence, in spite of a number of major simplifications and assumptions which are stated prior to the discussion, the laser analysis of the velocity, and hence the pressure distribution, supports the findings from the bucket analysis of the hydrofoils. 


\section{CONCLUSIONS}

An experimental study was carried out to investigate flow characteristics of alternative 2-D blade sections of rectangular planform, one of which was New Blade Section (NBS) developed in Newcastle University and other was well known NACA section. The tests involved the cavitation observations and the measurements of the local velocity distributions around the blade sections by using a 2-D LDA system. From the two sets of major tests the following conclusions are found:

- The cavitation tests of the two hydrofoils indicated that the onset of the cavitation was always observed at the leading edge. At negative angles of attack and small positive angles of attack up to about $1^{\circ}$, a pressure side cavitation was observed while at angles greater than $1^{\circ}$ suction side cavitation was observed.

- The pressure distribution analysis around the two alternative blade sections supported the analysis of the cavitation tests based on observations such that the both hydrofoils displayed strong suction peaks at the leading edge regions. Pressure peaks of the hydrofoils at the leading edge region are comparable. Therefore a slightly larger value of NBS pressure peak would onset the inception and hence narrower bucket arms for the NBS.

- In terms of the local pressure distribution, the fundamental difference between the two sections is that the NBS with sword like forward entry caused by the shift of the position of the maximum thickness towards the trailing edge presents lesser suction effect approximately along the forward half of the hydrofoil compared to the NACA. Whereas, the NACA hydrofoil, which has its maximum thickness at the mid-chord, displays lesser suction effect along the after half of the hydrofoil compared to the NBS.

- The method used to develop the NBS section did not predict tip vortex cavitation well and resulting in the failure of the NBS section for the propeller application. The method should use or investigate a proper tip vortex cavitation model for the prediction in order to obtain better section design.

- One can suggest that overall pressure distribution of the NBS section appears to be more sensitive to cavitation compared to the NACA section. This can be closely related to position of maximum thickness and its backward distribution as well as the fundamental shape of very fine leading edge. Therefore a further investigation into the modification of the thickness distribution and leading edge should be considered to improve cavitation behaviour of the NBS hydrofoil.

\section{ACKNOWLEDGEMENTS}

This study was supported by Sumitomo Heavy Industries, Japan. The Authors gratefully acknowledge the contributions from Dr. Sasaki of former member of Sumitomo Heavy Ind. and Mr I. Paterson, who was the supervisor of the Emerson Cavitation Tunnel, during the tests. The Authors also thank to Prof. B. Okan of Istanbul Technical University for his comments on the preparation of the paper.

\section{REFERENCES}

ASME, 1998. Test uncertainty: instruments and apparatus. ASME PTC 19.1, New Jersey, USA 31 December 1998.

Atlar, M., 2000. A history of the Emerson cavitation tunnel and its role in propeller cavitation research. Proceedings of International Conference on Propeller Cavitation NCT'50. Newcastle upon Tyne 3-5 April 2000, UK: Penshaw Press, pp.3-34.

Atlar, M. and Korkut, E., 1997. Emerson cavitation tunnel inflow characteristics based on 2-D laser Doppler anemometry analysis. Department of Marine Technology, University of Newcastle upon Tyne. Report No: MT-1997-001.

Atlar, M., Korkut, E., Wang, D. and Paterson, I., 1997. An investigation into cavitation inception characteristics of $2 D$ alternative blade sections by using Laser Doppler Anemometry. Department of Marine Technology, University of Newcastle upon Tyne. Report No: MT-1997-007.

Crompton, M.J. and Barrett, R.V., 2000. Investigation for the separation bubble formed behind sharp leading edge of a flat plate at incidence. Proceedings of the Institution of Mechanical Engineers, Part G: Journal of Aerospace Engineering. 214(March1), pp.157-176.

Dang, J., 2004. Improving cavitation performance with new blade sections for marine propellers. International Shipbuilding Progress, 51(4), pp.353-376. 
Dang, J., Tang, H., Cheng, J.D., Peng, X.X., Qin, Q. and Pan, S.S., 1998. New blade section design and its application. Proceedings of 3rd International Symposium on Cavitation, Cavitation'98. Grenoble, France April 1998, pp.301-308.

Eppler, R. and Somers, D.M., 1980. Computer program for the design and analysis of low speed airfoils. NASA Technical Memorandum, n.80210, 142 pages.

Eppler, R. and Shen, Y.T., 1979. Wings sections for hydrofoils-part 1: symmetrical profiles. Journal of Ship Research, 23(3), pp.209-217.

ITTC, 1990. Propulsor committee report. Proceedings of 19th International Towing Tank Conference. Madrid, Spain 16-22 September 1990.

ITTC, 1999. Propulsor committee report. Proceedings of 22nd International Towing Tank Conference. Seoul, Korea \& Shangai, China 5-11 September 1999.

Jessup, S.D., Berberich, W. and Remmers, K.D., 1994. Cavitation performance analysis of naval surface ship propellers with standard and new blade sections. Proceedings of the 20th Symposium on Naval Hydrodynamics. Santa Barbara, California, USA 21-26 August 1994.

Kawakita, C. and Hoshino, T., 1998. Design system of marine propellers with new blade sections. Proceedings of the 22nd Symposium on Naval Hydrodynamics. Washington D.C., USA 9-14 August 1994, pp.110-126.

Lee, J.-T., kim, M.C., Ahn, J.W. and Kim, H.C., 1993. Development of KD-propeller series using a new blade section. Transactions of the Society of Naval Architects of Korea, 28(2), pp.52-68.

Li, J.-H., Tang, D.-H. and Dong, S.-T., 2010. Propeller design by prescribed pressure distribution. Journal of Ship Mechanics, 14(1-2), pp.10-19.

Schlichting, H. and Gersten, K., 2000. Boundary-layer theory. 8th ed. Springer.

Wang, D., 1995. The development and validation of propeller design methods incorporating new approaches to blade section design. PhD. University of Newcastle upon Tyne.

Wang, D., Atlar, M. and Korkut, E., 1995. A propeller design method based on cavitation free buckets of blade sections. Proceedings of International Conference on Problems of Marine Propulsion, HYDRONAV'95. Gdansk, Poland 22-24 November 1995, pp.217-226.

Yamaguchi, H., Kato, H., Kamijo, A. and Maeda, M., 1988a. Development of marine propellers with better cavitation performance (2nd Report: Effect of design lift coefficient for propellers with flat pressure distribution). Journal of Society of Naval Architects of Japan, 163(6), pp.48-65.

Yamaguchi, H., Kato, H., Sugatani, A., Kamijo, A., Honda, T. and Maeda, M., 1988b. Development of marine propellers with better cavitation performance (3rd Report: Pressure distribution to stabilize cavitation). Journal of Society of Naval Architects of Japan, 164, pp.489-491.

Zhou, W.-X., Wu, Y.-H. and Dong, S.-T., 2001. A propeller design method with new blade section for improving cavitation inception under unsteady condition. Journal of Ship Mechanics, 5, pp.18-26. 